\title{
Supramolecular Allosteric Cofacial Porphyrin Complexes
}

\author{
Christopher G. Oliveri ${ }^{\dagger}$, Nathan C. Gianneschi ${ }^{\dagger}$, SonBinh T. Nguyen ${ }^{\star}, \dagger$, Chad A. Mirkin ${ }^{*}, \dagger$, \\ Charlotte L. Stern $\dagger$, Zdzislaw Wawrzak $\ddagger$, and Maren Pink $\S$ \\ Contribution from the Department of Chemistry and the International Institute for Nanotechnology, \\ Northwestern University, 2145 Sheridan Road, Evanston, Illinois, 60208-3113, Department of \\ Biochemistry, Molecular Biology and Cell Biology, Northwestern University, 2205 Tech Drive, \\ Evanston, Illinois 60208-3500, and Department of Chemistry, Indiana University, Bloomington, \\ Indiana 47405
}

\section{Abstract}

Nature routinely uses cooperative interactions to regulate cellular activity. For years, chemists have designed synthetic systems that aim toward harnessing the reactivity common to natural biological systems. By learning how to control these interactions in situ, one begins to allow for the preparation of man-made biomimetic systems that can efficiently mimic the interactions found in Nature. To this end, we have designed a synthetic protocol for the preparation of flexible metal-directed supramolecular cofacial porphyrin complexes which are readily obtained in greater than $90 \%$ yield through the use of new hemilabile porphyrin ligands with bifunctional ether-phosphine or thioetherphosphine substituents at the 5 and 15 positions on the porphyrin ring. The resulting architectures contain two hemilabile ligand-metal domains $\left(\mathrm{Rh}^{\mathrm{I}}\right.$ or $\mathrm{Cu}^{\mathrm{I}}$ sites) and two cofacially aligned porphyrins $\left(\mathrm{Zn}{ }^{\mathrm{II}}\right.$ sites), offering orthogonal functionalities and allowing these multimetallic complexes to exist in two states, "condensed" or "open". Combining the ether-phosphine ligand with the appropriate $\mathrm{Rh}^{\mathrm{I}}$ or $\mathrm{Cu}^{\mathrm{I}}$ transition-metal precursors results in "open" macrocyclic products. In contrast, reacting the thioether-phosphine ligand with $\mathrm{Rh}^{\mathrm{I}}$ or $\mathrm{Cu}^{\mathrm{I}}$ precursors yields condensed structures that can be converted into their "open" macrocyclic forms via introduction of additional ancillary ligands. The change in cavity size that occurs allows these structures to function as allosteric catalysts for the acyl transfer reaction between $X$-pyridylcarbinol (where $X=2,3$, or 4) and 1-acetylimidazole. For 3-and 4-pyridylcarbinol, the "open" macrocycle accelerates the acyl transfer reaction more than the condensed analogue and significantly more than the porphyrin monomer. In contrast, an allosteric effect was not observed for 2-pyridylcarbinol, which is expected to be a weaker binder and is unfavorably constrained inside the macrocyclic cavity.

\section{Introduction}

Over the past two decades, chemists have been designing synthetic systems that incorporate cofacial porphyrin entities. $1-10$ These systems are of particular interest owing to their unique photophysical properties, ${ }^{11-15}$ their ability to catalyze small molecule transformations, ${ }^{16-}$ 20 and their use in molecular recognition. ${ }^{21,22}$ Among these, cofacial porphyrin compounds exhibit unique properties attributed to their spatial arrangement with respect to each other, and therefore, the ability to control that arrangement via incorporation of multiple pophyrins in supramolecular arrays offers a potentially facile route to biomimetic structures with novel physicochemical properties. ${ }^{23,24}$ Taking inspiration from nature, a primary goal of our

\footnotetext{
E-mail: stn@northwestern.edu; chadnano@northwestern.edu.

Department of Chemistry and the International Institute for Nanotechnology, Northwestern University.

Department of Biochemistry, Molecular Biology and Cell Biology, Northwestern University.

§Indiana University.
} 
research has been to prepare supramolecular structures that exhibit allosteric behavior in the context of catalysis and small-molecule/ion sensing analgous to that displayed ubiquitously in natural enzymatic regulation. ${ }^{25-28}$ In this context, we reasoned that the ability to manipulate the distance between porphyrins by altering the shape and size of supramolecular cavities in which they reside, via external stimuli, may provide a means for designing abiotic allosteric mimics of biological systems. Indeed, a clear inspiration for this kind of allosteric control over the function of multi-porphyrin assemblies comes from perhaps the most famous example of allosteric control in biology, the cooperative homoallosteric protein hemoglobin, in which $\mathrm{O}_{2}$ binding at one subunit initiates conformational changes that facilitate subsequent $\mathrm{O}_{2}$ binding at other porphyrin domains. ${ }^{29}$

To design synthetic allosteric porphyrin-based structures, an attractive strategy entails the preparation of structures that provide facile control over both the orientation and distance of both porphyrins in the context of a small molecule-mediated reaction. To date, most approaches for the synthesis of cofacial porphyrins rely on the use of covalently attached organic molecules as spacers between the porphyrin units. $6,9,21,30-32$ As cofacial porphyrins have been prepared predominantly through synthetic organic approaches, only a few examples exist in which transition metals have been incorporated to facilitate the formation of cofacial porphyrins. ${ }^{24,33-36}$ Additionally, the backbones used to couple these cofacial porphyrins are often based upon rigid linker molecules, which restrict the overall flexibility of the molecule and make the incorporation of different substrates with varying size and shape challenging. To address this issue, several cofacial porphyrin structures have been designed which incorporate flexible linkages. ${ }^{22,37-40}$ However, few are able to specifically access a particular conformation via the introduction or removal of small-molecule effectors at a distal site (i.e., in an allosteric fashion). To this end, we hypothesized that coordination complexes of type $\mathbf{I}$ and II (Scheme 1), whose cavity size can be modified in situ with small molecules to form II would be very useful for designing stimulant-responsive porphyrin-based biomimetic systems.

Herein, we present a general, high-yielding synthetic methodology for the synthesis of cofacial porphyrin complexes that utilizes flexible porphyrin-based hemilabile ligands and $\mathrm{M}^{\mathrm{I}}$ precursors (where $\mathrm{M}^{\mathrm{I}}=\mathrm{Rh}^{\mathrm{I}}$ or $\mathrm{Cu}^{\mathrm{I}}$ ) to form macrocyclic products that can be chemically stimulated to change shape. Our strategy, based upon the Weak-Link Approach (WLA), ${ }^{41-}$ 43 provides rapid and convergent access to unique cofacial porphyrin systems because it relies on the use of metal-heteroatom interactions to facilitate the formation of the desired macrocyclic structures, thus allowing for the controlled and selective modification of the resulting structures in situ via introduction of specific external stimuli. Such stimulation can be used to tune the efficiency of a distance-dependent bimolecular acyl transfer reaction and discriminate geometric isomers of substituted pyridylcarbinol substrates.

\section{General Methods and Instrument Details}

All reactions were carried out under an inert atmosphere of nitrogen using standard Schlenk techniques or an inert atmosphere glovebox unless otherwise noted. Tetrahydrofuran (THF), diethyl ether $\left(\mathrm{Et}_{2} \mathrm{O}\right)$, dichloromethane $\left(\mathrm{CH}_{2} \mathrm{Cl}_{2}\right)$, acetonitrile $\left(\mathrm{CH}_{3} \mathrm{CN}\right)$, and hexanes were purified according to published methods. ${ }^{44}$ All solvents were deoxygenated with nitrogen or argon prior to use. 1-Chloro-2-diphenylphos-phinoethane (Organometallics Inc.), deuterated solvents (Cambridge Isotope Laboratories Inc.), $[\mathrm{Rh}(\mathrm{NBD}) \mathrm{Cl}]_{2}(\mathrm{NBD}=$ norbornadiene, Strem Chemicals), and 4-bromothiophenol (Alfa Aesar) were obtained from commercial sources and used as received. 4-(2-Chloroethoxy)-benzaldehyde, ${ }^{45}$ 2-(4-bromophenylsulfanyl) ethyldiphenylphosphane, ${ }^{46} 5$-mesityldipyrromethane, ${ }^{47}$ and (2-(mesitylthio)ethyl) diphenylphosphine 48 were prepared according to literature procedures. The synthesis of $[5,10,15,20$-tetraphenylporphyrinato $] z_{\text {inc }}{ }^{\mathrm{II}}(\mathrm{Zn}(\mathrm{TPP}))^{49}$ was adapted from a literature 
synthetic procedure. All other chemicals were used as received from Aldrich Chemical Co. ${ }^{1} \mathrm{H}$ NMR $(300.22 \mathrm{MHz})$ and ${ }^{13} \mathrm{C}\left\{{ }^{1} \mathrm{H}\right\}$ NMR $(75.50 \mathrm{MHz})$ spectra were recorded on a Varian Mercury $300 \mathrm{MHz}$ FT-NMR spectrometer and referenced relative residual proton resonances. ${ }^{31} \mathrm{P}\left\{{ }^{1} \mathrm{H}\right\}$ NMR $(121.53 \mathrm{MHz})$ spectra were recorded on a Varian Mercury 300 MHz FT-NMR spectrometer and referenced relative to an external $85 \% \mathrm{H}_{3} \mathrm{PO}_{4}$ standard. All chemical shifts are reported in ppm. Electrospray ionization mass spectra (ESIMS) were recorded on a Micromass Quatro II triple quadrapole mass spectrometer or a Micromass QTof Ultima mass spectrometer. Electron impact mass spectra (EIMS) were recorded on a Fisions VG 70-250 SE mass spectrometer. Elemental analyses were performed by Quantitative Technologies Inc., Whitehouse, NJ. Gas chromatography (GC) analyses of reaction mixtures were carried out on a computer-interfaced Agilent Technologies 6890 Network instrument equipped with a flame ionization detector (FID). The column used was a 30-m HP-5 capillary column with a $0.32-\mathrm{mm}$ inner diameter and a $0.25-\mu \mathrm{m}$ film thickness. GC yields were determined through integration of the product peak against biphenyl (internal standard) using pre-established response factors. GC retention times of products were confirmed with analytically pure samples.

\section{2-[4-(2-Chloroethoxy)-phenyl]-[1,3]dithiane (1)}

Under ambient conditions, 4-(2-chloroethoxy)benzaldehyde (3.00 g, $16.3 \mathrm{mmol})$ and 1,3propanedithiol $(1.95 \mathrm{~mL}, 19.2 \mathrm{mmol})$ were combined with $\mathrm{CH}_{3}-\mathrm{CN}(100 \mathrm{~mL})$ in a $250-\mathrm{mL}$ round-bottom flask and allowed to stir at room temperature for $5 \mathrm{~min}$ at which point $\mathrm{Y}$ $(\mathrm{OTf})_{3}(437 \mathrm{mg}, 5 \mathrm{~mol} \%$ ) was added. The resulting solution was stirred for another $30 \mathrm{~min}$ when it gradually became turbid due to product precipitation. The mixture was dried on a rotary evaporator to give an oily residue which was then dissolved in $\mathrm{CH}_{2} \mathrm{Cl}_{2}(100 \mathrm{~mL})$, washed with $\mathrm{H}_{2} \mathrm{O}(2 \times 25 \mathrm{~mL})$, dried over $\mathrm{MgSO}_{4}$, and concentrated in vacuo. The crude product was purified via column chromatography $\left(1: 1 \mathrm{v} / \mathrm{v}, \mathrm{CH}_{2} \mathrm{Cl}_{2} /\right.$ hexanes as an eluent) to yield $\mathbf{1}$ as a white solid (3.38 g, 75\% yield). ${ }^{1} \mathrm{H}$ NMR $\left(\mathrm{CD}_{2} \mathrm{Cl}_{2}\right): \delta 1.80\left(\mathrm{~m}, 1 \mathrm{H}, \mathrm{CH}_{2}-\mathrm{CH}_{A} \mathrm{H}_{\mathrm{B}}-\mathrm{CH}_{2}\right), 2.14$ (m, $\left.1 \mathrm{H}, \mathrm{CH}_{2}-\mathrm{CH}_{\mathrm{A}} \mathrm{H}_{B}-\mathrm{CH}_{2}\right), 2.86\left(\mathrm{~m}, 2 \mathrm{H}, \mathrm{SCH} \mathrm{H}_{2}\right), 2.92\left(\mathrm{~m}, 2 \mathrm{H}, \mathrm{SCH} \mathrm{CH}_{2}\right), 3.81\left(\mathrm{t}, 2 \mathrm{H}, \mathrm{CH}_{2} \mathrm{Cl}\right)$, $4.21\left(\mathrm{t}, 2 \mathrm{H}, \mathrm{OCH}_{2}\right), 5.15(\mathrm{~s}, 1 \mathrm{H}, \mathrm{S}-\mathrm{CH}-\mathrm{S}), 6.87\left(\mathrm{~d}, 2 \mathrm{H}, J_{\mathrm{H}-\mathrm{H}}=8.4 \mathrm{~Hz}, \mathrm{ArH}\right), 7.37(\mathrm{~d}, 2 \mathrm{H}$, $\left.J_{\mathrm{H}-\mathrm{H}}=8.7 \mathrm{~Hz}, \mathrm{ArH}\right) .{ }^{13} \mathrm{C}\left\{{ }^{1} \mathrm{H}\right\} \mathrm{NMR}\left(\mathrm{CDCl}_{3}\right): \delta 25.2\left(\mathrm{CH}_{2}-\mathrm{CH}_{2}-\mathrm{CH}_{2}\right), 32.4\left(\mathrm{CH}_{2}-\mathrm{CH}_{2}-\right.$ $\left.\mathrm{CH}_{2}\right), 42.0\left(\mathrm{ClCH}_{2}\right), 50.8(\mathrm{~S}-\mathrm{CH}-\mathrm{S}), 68.2\left(\mathrm{OCH}_{2}\right), 115.0(\mathrm{ArC}), 129.3(\mathrm{ArC}), 132.3$ $\left(\mathrm{CHC}_{\mathrm{ipso}}\right), 158.3\left(\mathrm{OC}_{\mathrm{ipso}}\right)$. EIMS $(\mathrm{m} / \mathrm{z})$ : Calcd. $274.03[\mathrm{M}]^{+}$. Found: 274.02. Elemental analysis for $\mathrm{C}_{12} \mathrm{H}_{15} \mathrm{ClOS}_{2}$ : Calcd. C, 52.44; H, 5.50. Found: C, 52.35; H, 5.44.

\section{[2-(4-[1,3]Dithian-2-yl-phenoxy)ethyl]diphenylphosphane (2)}

In a 100-mL Schlenk round-bottom flask, $1(1.00 \mathrm{~g}, 3.64 \mathrm{mmol})$ was dissolved in THF (50 $\mathrm{mL})$. To this solution, $\mathrm{KPPh}_{2}(7.28 \mathrm{~mL}$ of a $0.5 \mathrm{M}$ solution in THF, $3.64 \mathrm{mmol})$ was added over $10 \mathrm{~min}$ and allowed to stir for an additional $30 \mathrm{~min}$. The solvent was removed, and the residue was extracted with degassed $\mathrm{CH}_{2} \mathrm{Cl}_{2} / \mathrm{H}_{2} \mathrm{O}$. The solvent was removed from the organic fraction, yielding 2 as an off-white solid, which was recrystallized from $\mathrm{CH}_{2} \mathrm{Cl}_{2} /$ hexanes $(1.37$ $\mathrm{g}, 86 \%$ yield). ${ }^{1} \mathrm{H}$ NMR $\left(\mathrm{CD}_{2} \mathrm{Cl}_{2}\right): \delta 1.83\left(\mathrm{~m}, 1 \mathrm{H}, \mathrm{CH}_{2}-\mathrm{CH}_{A} \mathrm{H}_{\mathrm{B}}-\mathrm{CH}_{2}\right), 2.11\left(\mathrm{~m}, 1 \mathrm{H}, \mathrm{CH}_{2}-\right.$ $\left.\left.\mathrm{CH}_{\mathrm{A}} \mathrm{H}_{3}-\mathrm{CH}_{2}\right), 2.54\left(\mathrm{t}, 2 \mathrm{H}, \mathrm{CH}_{2} \mathrm{P}\right), 2.84\left(\mathrm{~m}, 2 \mathrm{H}, \mathrm{SCH}_{2}\right), 3.01(\mathrm{~m}, 2 \mathrm{H}, \mathrm{SCH})_{2}\right), 4.07(\mathrm{q}, 2 \mathrm{H}$, $\left.\mathrm{OCH}_{2}\right), 5.12(\mathrm{~s}, 1 \mathrm{H}, \mathrm{S}-\mathrm{CH}-\mathrm{S}), 6.73\left(\mathrm{~d}, 2 \mathrm{H}, J_{\mathrm{H}-\mathrm{H}}=8.7 \mathrm{~Hz}, \mathrm{ArH}\right), 7.31-7.50(\mathrm{br} \mathrm{m}, 12 \mathrm{H}, \mathrm{P}(\mathrm{Ar}-$ $H)) .{ }^{13} \mathrm{C}\left\{{ }^{1} \mathrm{H}\right\} \mathrm{NMR}\left(\mathrm{CD}_{2} \mathrm{Cl}_{2}\right): \delta 25.3\left(\mathrm{CH}_{2}-\mathrm{CH}_{2}-\mathrm{CH}_{2}\right), 28.3\left(\mathrm{CH}_{2}-\mathrm{CH}_{2}-\mathrm{CH}_{2}\right), 28.5\left(\mathrm{CH}_{2}-\right.$ $\left.\mathrm{CH}_{2}-\mathrm{CH}_{2}\right), 32.3\left(\mathrm{CH}_{2} \mathrm{P}\right), 50.8(\mathrm{~S}-\mathrm{CH}-\mathrm{S}), 65.5,65.8,114.8(\mathrm{ArC}), 128.7(\mathrm{ArC}), 128.8$ $(\operatorname{ArC}), 129.0(\operatorname{ArC}), 129.0(\operatorname{ArC}), 131.9(\operatorname{ArC}), 132.7(\operatorname{ArC}), 133.0(\operatorname{ArC}), 138.3(\operatorname{ArC}), 138.4$ $\left(\mathrm{CHC}_{\mathrm{ipso}}\right), 158.7\left(\mathrm{OC}_{\mathrm{ipso}}\right) .{ }^{31} \mathrm{P}\left\{{ }^{1} \mathrm{H}\right\}$ NMR $\left(\mathrm{CD}_{2} \mathrm{Cl}_{2}\right): \delta-21.6(\mathrm{~s})$. EIMS $(\mathrm{m} / \mathrm{z})$ : Calcd. 421.11 $[\mathrm{M}]^{+}$. Found: 421.10. Elemental analysis for $\mathrm{C}_{24} \mathrm{H}_{25} \mathrm{OPS}_{2}$ : Calcd. C, 67.90; H, 5.94. Found: C, 67.02; H, 5.86. 


\section{2-\{4-[2-(Diphenylphosphinothioyl)ethoxy]phenyl\}-(1,3)dithiane (3)}

In a 100-mL Schlenk round-bottom flask, $2(1.18 \mathrm{~g}, 2.76 \mathrm{mmol})$ and elemental sulfur (88.5 $\mathrm{mg}, 2.76 \mathrm{mmol})$ were stirred in THF $(50 \mathrm{~mL})$ for $4 \mathrm{~h}$ at which point the solvent was removed in vacuo and recrystallized from $\mathrm{CH}_{2} \mathrm{Cl}_{2}$ /hexanes to yield $\mathbf{3}$ as a light-yellow microcrystalline solid (1.23 g, 97\% yield). ${ }^{1} \mathrm{H}$ NMR $\left(\mathrm{CD}_{2} \mathrm{Cl}_{2}\right): \delta 1.83\left(\mathrm{~m}, 1 \mathrm{H}, \mathrm{CH}_{2}-\mathrm{CH}_{A} \mathrm{H}_{\mathrm{B}}-\mathrm{CH}_{2}\right), 2.11(\mathrm{~m}$, $\left.1 \mathrm{H}, \mathrm{CH}_{2}-\mathrm{CH}_{\mathrm{A}} \mathrm{H}_{B}-\mathrm{CH}_{2}\right), 2.84\left(\mathrm{~m}, 6 \mathrm{H},\left(\mathrm{SCH}_{2}\right)_{2}\right.$ and $\left.\mathrm{CH}_{2} \mathrm{P}\right), 4.35\left(\mathrm{~m}, 2 \mathrm{H}, \mathrm{OCH}_{2}\right), 5.11(\mathrm{~s}, 1 \mathrm{H}$, $\mathrm{S}-\mathrm{CH}-\mathrm{S}), 6.62\left(\mathrm{~d}, 2 \mathrm{H}, J_{\mathrm{H}-\mathrm{H}}=8.7 \mathrm{~Hz}, \mathrm{ArH}\right), 7.27\left(\mathrm{~d}, 2 \mathrm{H}, J_{\mathrm{H}-\mathrm{H}}=8.4 \mathrm{~Hz}, \mathrm{ArH}\right), 7.46-7.91(\mathrm{br}$ $\mathrm{m}, 10 \mathrm{H}, \mathrm{P}(\mathrm{ArH})) .{ }^{13} \mathrm{C}\left\{{ }^{1} \mathrm{H}\right\} \mathrm{NMR}\left(\mathrm{CDCl}_{3}\right): \delta 25.2\left(\mathrm{CH}_{2}-\mathrm{CH}_{2}-\mathrm{CH}_{2}\right), 32.4\left(\mathrm{CH}_{2}-\mathrm{CH}_{2}-\mathrm{CH}_{2}\right)$, $33.0\left(\mathrm{CH}_{2}-\mathrm{CH}_{2}-\mathrm{CH}_{2}\right), 50.9\left(\mathrm{CH}_{2} \mathrm{P}=\mathrm{S}\right), 53.6(\mathrm{~S}-\mathrm{CH}-\mathrm{S}), 62.5\left(\mathrm{OCH}_{2}\right), 114.8(\mathrm{ArC}), 128.8$ $(\operatorname{ArC}), 129.0(\operatorname{ArC}), 129.1(\operatorname{ArC}), 131.1(\operatorname{ArC}), 131.3(\operatorname{ArC}), 131.8(\operatorname{ArC}), 131.9(\operatorname{ArC}), 132.2$ $(\mathrm{ArC}), 133.3(\mathrm{ArC}), 158.1\left(\mathrm{OC}_{\mathrm{ipso}}\right) .{ }^{31} \mathrm{P}-\left\{{ }^{1} \mathrm{H}\right\}$ NMR $\left(\mathrm{CD}_{2} \mathrm{Cl}_{2}\right): \delta 39.3(\mathrm{~s})$. EIMS $(\mathrm{m} / \mathrm{z})$ : Calcd. $456.08\left[\mathrm{M}^{+}\right]$. Found. 456.07. Elemental analysis for $\mathrm{C}_{24} \mathrm{H}_{25} \mathrm{OPS}_{3}$ : Calcd. C, 68.84; H, 5.23. Found: C, 67.43; H, 4.90 .

\section{4-[2-(Diphenylphosphinothioyl)ethoxy]benzaldehyde (4)}

Under ambient conditions, $\mathrm{NaNO}_{2}(226 \mathrm{mg}, 3.27 \mathrm{mmol})$ and acetyl chloride $(0.233 \mathrm{~mL}, 3.27$ mmol) were stirred in in $\mathrm{CH}_{2} \mathrm{Cl}_{2}(10 \mathrm{~mL})$ a $100-\mathrm{mL}$ round-bottom flask at $0{ }^{\circ} \mathrm{C}$ for $10 \mathrm{~min}$. A solution of $\mathbf{3}(500 \mathrm{mg}, 1.09 \mathrm{mmol})$ in $\mathrm{CH}_{2} \mathrm{Cl}_{2}$ was added and stirred for an additional $5 \mathrm{~min}$ at $0^{\circ} \mathrm{C}$. At this point, $\mathrm{H}_{2} \mathrm{O}(5 \mathrm{~mL})$ was added and the reaction was brought to room temperature and allowed to stir for an hour. The reaction was neutralized with a saturated aqueous solution of $\mathrm{NaHCO}_{3}$ and was extracted with $\mathrm{CH}_{2} \mathrm{Cl}_{2}(50 \mathrm{~mL})$. The organic layer was washed with $\mathrm{H}_{2} \mathrm{O}(2 \times 10 \mathrm{~mL})$ and dried over $\mathrm{MgSO}_{4}$. After removing the solvent in vacuo, the crude product was purified via column chromatography $(1: 1 \mathrm{v} / \mathrm{v}$, ethyl acetate/hexanes as eluent $)$ yielding 4 as a light-yellow microcrystalline solid ( $352 \mathrm{mg}, 88 \%$ yield). ${ }^{1} \mathrm{H} \mathrm{NMR}\left(\mathrm{CD}_{2} \mathrm{Cl}_{2}\right): \delta 2.98(\mathrm{~m}$, $\left.2 \mathrm{H}, \mathrm{CH}_{2} \mathrm{P}\right), 4.46\left(\mathrm{~m}, 2 \mathrm{H}, \mathrm{OCH}_{2}\right), 6.78\left(\mathrm{~d}, 2 \mathrm{H}, J_{\mathrm{H}-\mathrm{H}}=9 \mathrm{~Hz}, \mathrm{ArH}\right), 7.48-7.91(\mathrm{br} \mathrm{m}, 12 \mathrm{H}, \mathrm{P}$ $(\mathrm{Ar} H)), 9.84(\mathrm{~s}, 1 \mathrm{H}, \mathrm{CHO}) .{ }^{13} \mathrm{C}\left\{{ }^{1} \mathrm{H}\right\} \mathrm{NMR}\left(\mathrm{CD}_{2} \mathrm{Cl}_{2}\right): \delta 32.1$ (d, $\left.J_{\mathrm{C}-\mathrm{P}}=56 \mathrm{~Hz}, \mathrm{CH}_{2} \mathrm{P}=\mathrm{S}\right), 63.0$ $\left(\mathrm{OCH}_{2}\right), 114.8(\mathrm{ArC}), 128.8\left(C_{\mathrm{ipso}} \mathrm{CHO}\right), 128.9(\mathrm{ArC}), 130.4(\mathrm{ArC}), 131.1(\mathrm{ArC}), 131.2$ $(\operatorname{ArC}), 131.9(\operatorname{ArC}), 132.4(\operatorname{ArC}), 133.5(\operatorname{ArC}), 163.2\left(C_{\mathrm{ipso}} \mathrm{O}\right), 190.7(\mathrm{CHO}) .{ }^{31} \mathrm{P}\left\{{ }^{1} \mathrm{H}\right\} \mathrm{NMR}$ $\left(\mathrm{CD}_{2} \mathrm{Cl}_{2}\right): \delta 39.2(\mathrm{~s})$. EIMS $(\mathrm{m} / \mathrm{z})$ : Calcd. $366.08\left[\mathrm{M}^{+}\right]$. Found. 366.08. Elemental analysis for $\mathrm{C}_{21} \mathrm{H}_{19} \mathrm{O}_{2}$ PS: Calcd. C, 63.13; H, 5.52. Found: C, 62.68; H, 5.22.

\section{5,15-Bis-\{4-[2-(diphenylphosphinothioyl)ethoxy]phenyl\}-10,20-bis-(mesityl)porphyrin (5)}

In an aluminum-foil-wrapped 1000-mL Schlenk round-bottom flask, 4 (1.47 g, $4 \mathrm{mmol}), 5$ mesityldipyrromethane (1.06 g, $4 \mathrm{mmol})$, and activated molecular sieves $(4 \AA)$ were stirred in $\mathrm{CHCl}_{3}(600 \mathrm{~mL})$ and degassed under a stream of $\mathrm{N}_{2}$ for $15 \mathrm{~min} . \mathrm{BF}_{3} \cdot \mathrm{OEt}_{2}(0.420 \mathrm{~mL})$ was added dropwise to this solution, and the resulting mixture was allowed to stir for $3 \mathrm{~h}$ under $\mathrm{N}_{2}$. DDQ (1.09 g, $\left.4.8 \mathrm{mmol}\right)$ was then added as a solid under a stream of $\mathrm{N}_{2}$, and the reaction was allowed to stir for an additional $30 \mathrm{~min}$ at which point $\mathrm{NEt}_{3}(4 \mathrm{~mL})$ was added. The reaction mixture was stirred for $1 \mathrm{~min}$ before being filtered through a pad of Celite to remove the sieves. The solution was concentrated in vacuo and the resulting residue was dissolved in a minimum amount $\mathrm{CH}_{2} \mathrm{Cl}_{2}$ and poured on top of a silica gel column and purified via flash chromatography (eluent $\mathrm{CH}_{2} \mathrm{Cl}_{2}$ ) to yield 5 as a purple microcrystalline solid (1.01 g, $41 \%$ yield). ${ }^{1} \mathrm{H}$ NMR $\left(\mathrm{CD}_{2} \mathrm{Cl}_{2}\right): \delta-2.69(\mathrm{~s}, 2 \mathrm{H}, \mathrm{NH}), 1.82\left(\mathrm{~s}, 12 \mathrm{H}, \mathrm{CH}_{3}\right), 2.66\left(\mathrm{~s}, 6 \mathrm{H}, \mathrm{CH}_{3}\right), 3.16\left(\mathrm{~m}, 4 \mathrm{H}, \mathrm{CH}_{2} \mathrm{P}=\mathrm{S}\right)$, $4.66\left(\mathrm{~m}, 4 \mathrm{H}, \mathrm{OCH}_{2}\right), 7.07\left(\mathrm{~d}, 4 \mathrm{H}, J_{\mathrm{H}-\mathrm{H}}=7.8 \mathrm{~Hz}, \mathrm{ArH}\right), 7.30(\mathrm{~s}, 4 \mathrm{H}), 7.59(\mathrm{br} \mathrm{s}, 12 \mathrm{H}, \mathrm{ArH})$, $7.96(\mathrm{~m}, 12 \mathrm{H}, \operatorname{Ar} H), 8.65\left(\mathrm{~d}, 4 \mathrm{H}, J_{\mathrm{H}-\mathrm{H}}=5.1 \mathrm{~Hz}, \operatorname{Ar} H\right), 8.79\left(\mathrm{~d}, 4 \mathrm{H}, J_{\mathrm{H}-\mathrm{H}}=4.5 \mathrm{~Hz}\right.$,

$\operatorname{Ar} H) .{ }^{31} \mathrm{P}\left\{{ }^{1} \mathrm{H}\right\}$ NMR $\left(\mathrm{CD}_{2} \mathrm{Cl}_{2}\right): \delta 39.5(\mathrm{~s})$. ESIMS $(\mathrm{m} / z)$ : Calcd. $1219.4\left[\mathrm{M}^{+}\right]$. Found: 1219.3. Elemental analysis for $\mathrm{C}_{78} \mathrm{H}_{68} \mathrm{~N}_{4} \mathrm{O}_{2} \mathrm{P}_{2} \mathrm{~S}_{2}$ : Calcd. C, 76.82; H, 5.62; N, 4.59. Found: C, 76.04; $\mathrm{H}, 5.18 ; \mathrm{N}, 4.65$. 
[5,15-Bis-\{4-[2-(diphenylphosphinothioyl)ethoxy]phenyl\}-10,20-bis-(mesityl)porphyrinato] zinc (II) (6)

Under ambient conditions, $5(500 \mathrm{mg}, 0.399 \mathrm{mmol})$ and $\mathrm{Zn}(\mathrm{OAc})_{2} \cdot 2 \mathrm{H}_{2} \mathrm{O}(700 \mathrm{mg}, 3.19 \mathrm{mmol})$ were combined in a 500-mL round-bottom flask and stirred under reflux for $4 \mathrm{~h}$ in a $\mathrm{CHCl}_{3} /$ $\mathrm{CH}_{3} \mathrm{OH}(4: 1 \mathrm{v} / \mathrm{v}, 350 \mathrm{~mL})$ solution. The solution was then washed with $\mathrm{H}_{2} \mathrm{O}(100 \mathrm{~mL})$ and extracted with $\mathrm{CHCl}_{3}(2 \times 100 \mathrm{~mL})$. The organic layer was further washed with $\mathrm{H}_{2} \mathrm{O}(100 \mathrm{~mL})$, dried over $\mathrm{Na}_{2} \mathrm{SO}_{4}$, and concentrated to give 6 as a purple microcrystalline solid $(507 \mathrm{mg}, 96 \%$ yield). ${ }^{1} \mathrm{H}$ NMR (THF- $\left.d_{8}\right): \delta 1.83\left(\mathrm{~s}, 12 \mathrm{H}, \mathrm{CH}_{3}\right), 2.60\left(\mathrm{~m}, 6 \mathrm{H}, \mathrm{CH}_{3}\right), 2.79\left(\mathrm{~m}, 4 \mathrm{H}, \mathrm{CH}_{2} \mathrm{P}=\mathrm{S}\right)$, $4.36\left(\mathrm{~m}, 4 \mathrm{H}, \mathrm{OCH}_{2}\right), 7.16$ (br m, 28H, $\left.\mathrm{ArH}\right), 8.09\left(\mathrm{~d}, 4 \mathrm{H}, J_{\mathrm{H}-\mathrm{H}}=8.4 \mathrm{~Hz}, \mathrm{ArH}\right), 8.61(\mathrm{~d}, 4 \mathrm{H}$, $\left.J_{\mathrm{H}-\mathrm{H}}=4.5 \mathrm{~Hz}, \operatorname{Ar} H\right), 8.77\left(\mathrm{~d}, 4 \mathrm{H}, J_{\mathrm{H}-\mathrm{H}}=4.5 \mathrm{~Hz}, \operatorname{Ar} H\right) .{ }^{31} \mathrm{P}\left\{{ }^{1} \mathrm{H}\right\} \mathrm{NMR}\left(\mathrm{THF}-d_{8}\right): \delta 39.2(\mathrm{~s})$. $\operatorname{ESIMS~}(\mathrm{m} / \mathrm{z})$ : Calcd. $1282.8\left[\mathrm{M}^{+}\right]$. Found: 1282.2. Elemental analysis for $\mathrm{C}_{78} \mathrm{H}_{66} \mathrm{~N}_{4} \mathrm{O}_{2} \mathrm{P}_{2} \mathrm{~S}_{2} \mathrm{Zn}$ : Calcd. C, 73.03; H, $5.19 \mathrm{H} ; \mathrm{N}, 4.37$. Found: C, 72.89; H, 5.23; N, 4.17.

\section{[5,15-Bis-[4-(2-diphenylphosphanylethoxy)phenyl]-10,20-bis-(mesityl)porphyrinato]zinc(II)}

(7)

In a 50-mL Schlenk round-bottom flask, 6 (300 mg, $0.234 \mathrm{mmol})$ and $\mathrm{Cp}_{2} \mathrm{ZrHCl}(392 \mathrm{mg}, 1.52$ mmol) were stirred in THF under $\mathrm{N}_{2}(40 \mathrm{~mL})$ at $60{ }^{\circ} \mathrm{C}$ for $4 \mathrm{~h}$. The solvent was removed and the reaction was purified via column chromatography (silica gel, THF) in a glove box under an atmosphere of $\mathrm{N}_{2}$. The solvent was removed in vacuo to yield $\mathbf{7}$ as a purple microcrystalline solid (254 mg, 89\% yield). ${ }^{1} \mathrm{H}$ NMR (THF- $\left.d_{8}\right): \delta 1.83$ (s, $\left.12 \mathrm{H}, \mathrm{CH}_{3}\right), 2.60\left(\mathrm{~s}, 6 \mathrm{H}, \mathrm{CH}_{3}\right), 2.77$ $\left(\mathrm{m}, 4 \mathrm{H}, \mathrm{CH}_{2} \mathrm{P}\right), 4.36\left(\mathrm{~m}, 4 \mathrm{H}, \mathrm{CH}_{2} \mathrm{O}\right), 7.17\left(\mathrm{~d}, 4 \mathrm{H}, J_{\mathrm{H}-\mathrm{H}}=6.6 \mathrm{~Hz}, \mathrm{ArH}\right), 7.30-7.61(\mathrm{bm}, 24 \mathrm{H}$, $\operatorname{Ar} H), 8.01\left(\mathrm{~d}, 4 \mathrm{H}, J_{\mathrm{H}-\mathrm{H}}=8.7 \mathrm{~Hz}, \operatorname{Ar} H\right), 8.64\left(\mathrm{~d}, 4 \mathrm{H}, J_{\mathrm{H}-\mathrm{H}}=4.5 \mathrm{~Hz}, \mathrm{Ar} H\right), 8.78(\mathrm{~d}, 4 \mathrm{H}$, $\left.J_{\mathrm{H}-\mathrm{H}}=4.5 \mathrm{~Hz}, \operatorname{Ar} H\right) .{ }^{31} \mathrm{P}\left\{{ }^{1} \mathrm{H}\right\}$ NMR $\left(\mathrm{THF}-d_{8}\right): \delta-21.2(\mathrm{~s})$. ESIMS $(\mathrm{m} / z)$ : Calcd. 1218.74 $\left[\mathrm{M}^{+}\right]$. Found: 1217.4. Elemental analysis for $\mathrm{C}_{78} \mathrm{H}_{66} \mathrm{~N}_{4} \mathrm{O}_{2} \mathrm{P}_{2} \mathrm{Zn}$ : Calcd. C, 76.87; H, 5.46; N, 4.60. Found: C, 76.37; H, 5.18; N, 4.45.

\section{$[(7) \mathrm{RhCl}(\mathrm{CO})]_{2}$ Macrocycle (8a)}

A small vial was charged with $\left[\mathrm{Rh}(\mathrm{CO})_{2}(\mathrm{Cl})\right]_{2}(4.80 \mathrm{mg}, 0.0123 \mathrm{mmol})$ and $\mathrm{CH}_{2} \mathrm{Cl}_{2}(2 \mathrm{~mL})$. The resulting solution was stirred for $1 \mathrm{~min}$, at which point a solution of $7(30.0 \mathrm{mg}, 0.0246$ $\mathrm{mmol})$ in THF $(5 \mathrm{~mL})$ was added dropwise over $1 \mathrm{~min}$. The resulting solution was stirred for $3 \mathrm{~h}$. The solvent was removed and the product was recrystallized from $\mathrm{CH}_{2} \mathrm{Cl}_{2} /$ pentane ( 32.1 $\mathrm{mg}, 94 \%$ yield). ${ }^{1} \mathrm{H} \mathrm{NMR}\left(\mathrm{CD}_{2} \mathrm{Cl}_{2}\right): \delta 1.54\left(\mathrm{~s}, 24 \mathrm{H}, \mathrm{CH}_{3}\right), 2.56\left(\mathrm{~s}, 12 \mathrm{H}, \mathrm{CH}_{3}\right), 3.34$ (br m, $8 \mathrm{H}$, $\mathrm{CH}_{2} \mathrm{P}$ ), 4.81 (br m, 8H, CH $\mathrm{H}_{2} \mathrm{O}$ ), 7.10 (br m, 22H, $\mathrm{ArH}$ ), 7.47 (m, 22H, $\mathrm{ArH}$ ), 7.90 (br m, 20H, $\mathrm{Ar} H), 8.79$ (br m, $16 \mathrm{H}, \mathrm{Ar} H) .{ }^{31} \mathrm{P}\left\{{ }^{1} \mathrm{H}\right\}$ NMR $\left(\mathrm{CD}_{2} \mathrm{Cl}_{2}\right): \delta 21.6\left(\mathrm{~d}, J_{\mathrm{Rh}-\mathrm{P}}=124 \mathrm{~Hz}\right)$. ESIMS $(\mathrm{m} / z)$ for $\left[\mathrm{C}_{156} \mathrm{H}_{132} \mathrm{~N}_{8} \mathrm{O}_{4} \mathrm{P}_{4} \mathrm{Rh}_{2} \mathrm{Zn}_{2}(\mathrm{CO})_{2}\right]^{2+}$ : Calcd. 1349.6. Found: 1349.3. Elemental analysis for $\mathrm{C}_{158} \mathrm{H}_{132} \mathrm{Cl}_{2} \mathrm{~N}_{8} \mathrm{O}_{6} \mathrm{P}_{4} \mathrm{Rh}_{2} \mathrm{Zn}_{2}$ : Calcd. C, 68.51; H, $4.80 \mathrm{H} ; \mathrm{N}$, 4.04. Found: C, 68.38; $\mathrm{H}, 4.91 ; \mathrm{N}, 3.52$.

\section{$\left[(7) \mathrm{Cu}\left(\mathrm{CH}_{3} \mathrm{CN}\right)_{2}\left(\mathrm{PF}_{6}\right)\right]_{2}$ Macrocycle (8b)}

A 50-mL Schlenk flask was charged with $\left[\mathrm{Cu}\left(\mathrm{CH}_{3} \mathrm{CN}\right)_{4}\right] \mathrm{PF}_{6}(29.8 \mathrm{mg}, 0.0799 \mathrm{mmol})$ and $\mathrm{CH}_{2}-\mathrm{Cl}_{2}(5 \mathrm{~mL})$. A THF solution of 7 (100 mg, $0.0799 \mathrm{mmol}, 20 \mathrm{~mL}$ ) was added to the " $\mathrm{Cu}$ " solution dropwise over $5 \mathrm{~min}$ at room temperature to give a red/purple solution, which was then allowed to stir for $3 \mathrm{~h}$. The solvent was removed to yield a purple microcrystalline solid which was recrystallized from $\mathrm{CH}_{2} \mathrm{Cl}_{2} /$ pentane $\left(107 \mathrm{mg}\right.$, $92 \%$ yield). ${ }^{1} \mathrm{H} \mathrm{NMR}\left(\mathrm{CD}_{2} \mathrm{Cl}_{2}\right): \delta$ 1.66 (s, 24H, $\mathrm{CH}_{3}$ ), 2.07 (s, 12H, $\mathrm{CH}_{3} \mathrm{CN}$ ), 2.43 (s, 12H, $\mathrm{CH}_{3}$ ), 2.95 (br m, 8H, $\mathrm{CH}_{2} \mathrm{P}$ ), 4.46 (br m, 8H, $\mathrm{CH}_{2} \mathrm{O}$ ), $7.10(\mathrm{~s}, 22 \mathrm{H}, \mathrm{ArH}), 7.50(\mathrm{~s}, 22 \mathrm{H}, \mathrm{ArH}), 7.62(12 \mathrm{H}, \mathrm{ArH}), 8.00(\mathrm{~d}, 8 \mathrm{H}$, $\left.J_{\mathrm{H}-\mathrm{H}}=7.8 \mathrm{~Hz}, \operatorname{Ar} H\right), 8.64\left(\mathrm{~d}, 8 \mathrm{H}, J_{\mathrm{H}-\mathrm{H}}=4.2 \mathrm{~Hz}, \operatorname{Ar} H\right), 8.78\left(\mathrm{~d}, 8 \mathrm{H}, J_{\mathrm{H}-\mathrm{H}}=4.2 \mathrm{~Hz}\right.$, $\mathrm{Ar} H) .{ }^{31} \mathrm{P}\left\{{ }^{1} \mathrm{H}\right\}$ NMR $\left(\mathrm{CD}_{2} \mathrm{Cl}_{2}\right): \delta-11.5(\mathrm{~s})$. ESIMS $(\mathrm{m} / z)$ for $\left[\mathrm{C}_{156} \mathrm{H}_{132} \mathrm{~N}_{8} \mathrm{~S}_{4} \mathrm{P}_{4} \mathrm{Cu}_{2} \mathrm{Zn}_{2}\right]^{2+}$ : Calcd. 1282.2. Found: 1282.4. Elemental analysis for $\left[\mathrm{C}_{164} \mathrm{H}_{144} \mathrm{~N}_{12} \mathrm{O}_{4} \mathrm{P}_{6} \mathrm{~F}_{12} \mathrm{Zn}_{2} \mathrm{Cu}_{2}\right]$ : Calcd. C, 65.25; H, 4.81; N, 5.57. Found: C, 65.32; H, 4.56; N, 6.35. 


\section{1-Bromo-4-[2-(diphenylphosphinothioyl)ethylsulfanyl]-benzene (9)}

In a 100-mL Schlenk round-bottom flask, 2-(4-bromo-phenylsulfanyl)ethyldiphenylphosphane ( $3.00 \mathrm{~g}, 7.48 \mathrm{mmol})$, and elemental sulfur $(264 \mathrm{mg}, 8.22 \mathrm{mmol})$ were stirred in THF ( $150 \mathrm{~mL})$ under $\mathrm{N}_{2}$ for $3 \mathrm{~h}$. The reaction mixture was concentrated in vacuo, and the product was purified via column chromatography (1:1 v/v, $\mathrm{CH}_{2} \mathrm{Cl}_{2} /$ hexanes).

Compound 9 was isolated as an off-white microcrystalline solid (3.02 g, 92\% yield). ${ }^{1} \mathrm{H}$ NMR $\left(\mathrm{CD}_{2} \mathrm{Cl}_{2}\right): \delta 2.68\left(\mathrm{~m}, 2 \mathrm{H}, \mathrm{CH}_{2} \mathrm{P}=\mathrm{S}\right), 3.10\left(\mathrm{~m}, 2 \mathrm{H}, \mathrm{SCH}_{2}\right), 7.12\left(\mathrm{~d}, 2 \mathrm{H}, J_{\mathrm{H}-\mathrm{H}}=8.7 \mathrm{~Hz}, \mathrm{Ar} H\right)$, $7.39\left(\mathrm{~d}, 2 \mathrm{H}, J_{\mathrm{H}-\mathrm{H}}=8.7 \mathrm{~Hz}, \operatorname{Ar} H\right), 7.48-7.79(\mathrm{~m}, 10 \mathrm{H}, \mathrm{P}(\operatorname{Ar} H)) .{ }^{13} \mathrm{C}\left\{{ }^{1} \mathrm{H}\right\} \mathrm{NMR}\left(\mathrm{CDCl}_{3}\right): \delta$ $26.9\left(\mathrm{SCH}_{2}\right), 32.3\left(\mathrm{~d}, \mathrm{CH}_{2} \mathrm{P}=\mathrm{S}, J_{\mathrm{C}-\mathrm{P}}=51.3 \mathrm{~Hz}\right), 128.9(\mathrm{ArC}), 129.1(\mathrm{ArC}), 131.0(\mathrm{ArC}), 131.1$ $(\operatorname{ArC}), 131.2(\operatorname{ArC}), 131.6(\operatorname{ArC}), 132.0(\operatorname{ArC}), 132.3(\operatorname{ArC}) .{ }^{31} \mathrm{P}\left\{{ }^{1} \mathrm{H}\right\} \mathrm{NMR}\left(\mathrm{CD}_{2} \mathrm{Cl}_{2}\right): \delta 41.4$ (s). EIMS (m/z): Calcd. 431.97 [M+]. Found: 431.97. Elemental analysis for $\mathrm{C}_{20} \mathrm{H}_{18}-\mathrm{BrPS}_{2}$ : Calcd. C, 55.43; H, 4.19. Found: C, 55.59, H, 4.07.

\section{4-[2-(Diphenylphosphinothioyl)ethylsulfanyl]benzaldehyde (10)}

Compound $9(3.00 \mathrm{~g}, 6.90 \mathrm{mmol})$ was dissolved in THF $(60 \mathrm{~mL})$ in a 100-mL Schlenk roundbottom flask and was cooled to $-78{ }^{\circ} \mathrm{C} . n$-BuLi $(2.76 \mathrm{~mL}, 6.90 \mathrm{mmol}, 2.5 \mathrm{M}$ in hexanes) was added dropwise to the solution over $5 \mathrm{~min}$ and the mixture was allowed to stir for $30 \mathrm{~min}$, before DMF $(0.802 \mathrm{~mL}, 10.35 \mathrm{mmol})$ was added to the flask. This solution was cooled to -78 ${ }^{\circ} \mathrm{C}$ and allowed to stir for an additional $30 \mathrm{~min}$ before the temperature was allowed to rise back to room temperature. The mixture was quenched with $\mathrm{H}_{2} \mathrm{O}$ followed by extraction with $\mathrm{CH}_{2} \mathrm{Cl}_{2}$. The organic layer was dried over $\mathrm{MgSO}_{4}$, filtered, and concentrated in vacuo to give a yellow crude product which was then recrystallized $\left(\mathrm{CH}_{2} \mathrm{Cl}_{2} /\right.$ pentane) to yield $\mathbf{1 0}$ as a lightyellow microcrystalline solid $(2.35 \mathrm{~g}, 88 \%$ yield $) .{ }^{1} \mathrm{H} \mathrm{NMR}\left(\mathrm{CD}_{2} \mathrm{Cl}_{2}\right): \delta 2.76(\mathrm{~m}, 2 \mathrm{H}$, $\left.\mathrm{CH}_{2} \mathrm{P}=\mathrm{S}\right), 3.23\left(\mathrm{~m}, 2 \mathrm{H}, \mathrm{SCH} H_{2}\right), 7.29\left(\mathrm{~d}, 2 \mathrm{H}, J_{\mathrm{H}-\mathrm{H}}=6.6 \mathrm{~Hz}, \mathrm{ArH}\right) 7.47(\mathrm{~m}, 6 \mathrm{H}, \mathrm{P}(\mathrm{ArH})), 7.73$ $\left(\mathrm{d}, 2 \mathrm{H}, J_{\mathrm{H}-\mathrm{H}}=8.7 \mathrm{~Hz}, \operatorname{Ar} H\right), 7.79(\mathrm{~m}, 4 \mathrm{H}, \mathrm{P}(\mathrm{ArH})), 9.92(\mathrm{~s}, 1 \mathrm{H}, \mathrm{CHO}) .{ }^{13} \mathrm{C}\left\{{ }^{1} \mathrm{H}\right\} \mathrm{NMR}$ $\left(\mathrm{CDCl}_{3}\right): \delta 25.0\left(\mathrm{SCH}_{2}\right), 32.0\left(\mathrm{~d}, \mathrm{CH}_{2} \mathrm{P}=\mathrm{S}, J_{\mathrm{C}-\mathrm{P}}=51.3 \mathrm{~Hz}\right), 126.8(\mathrm{ArC}), 129.0(\mathrm{ArC}), 129.1$ $(\operatorname{ArC}), 130.4(\operatorname{ArC}), 131.1(\operatorname{ArC}), 131.3(\operatorname{ArC}), 132.1(\operatorname{ArC}), 132.5(\operatorname{ArC}), 191.4(C H O) .{ }^{31} \mathrm{P}$ $\left\{{ }^{1} \mathrm{H}\right\}$ NMR $\left(\mathrm{CD}_{2} \mathrm{Cl}_{2}\right): \delta 41.5$ (s). EIMS $(\mathrm{m} / \mathrm{z})$ : Calcd. $382.01\left[\mathrm{M}^{+}\right]$. Found. 382.06. Elemental analysis for $\mathrm{C}_{21} \mathrm{H}_{19}-\mathrm{OPS}_{2}$ : Calcd. C, 65.94; H, 5.01. Found: C, 65.23; H, 4.71.

\section{[5,15-Bis-[4-(2-diphenylphosphinothioylethylsulfanyl)phenyl]-10,-20-bis-(mesityl)porphyrin} (11)

In an aluminum-foil-wrapped 1000-mL Schlenk flask, compound 10 (1.23 g, $3.20 \mathrm{mmol})$, 5mesityldipyrromethane ( $848 \mathrm{mg}, 3.20 \mathrm{mmol}$ ), and activated molecular sieves ( $4 \AA$ ) were stirred in $\mathrm{CHCl}_{3}(600 \mathrm{~mL})$ and degassed under a stream of $\mathrm{N}_{2}$ for $15 \mathrm{~min} . \mathrm{BF}_{3} \cdot \mathrm{OEt}_{2}(0.350 \mathrm{~mL})$ was added dropwise to this solution, and the resulting mixture was allowed to stir for $3 \mathrm{~h}$ under $\mathrm{N}_{2}$. DDQ ( $862 \mathrm{mg}, 3.8 \mathrm{mmol}$ ) was then added as a solid under a stream of $\mathrm{N}_{2}$, and the reaction was allowed to stir for an additional $30 \mathrm{~min}$ at which point $\mathrm{NEt}_{3}(4 \mathrm{~mL})$ was added. The reaction mixture was stirred for $1 \mathrm{~min}$ before being filtered through a pad of Celite to remove the sieves. The solution was concentrated in vacuo and the resulting residue was dissolved in $\mathrm{CH}_{2} \mathrm{Cl}_{2}$ and poured on top of a silica gel column (eluent $\mathrm{CH}_{2} \mathrm{Cl}_{2}$ ). Subsequent chromatography yielded 11 as a purple microcrystalline solid ( $879 \mathrm{mg}, 44 \%$ yield). ${ }^{1} \mathrm{H}$ NMR $\left(\mathrm{CD}_{2} \mathrm{Cl}_{2}\right): \delta-2.67(\mathrm{~s}, 2 \mathrm{H}$, $\mathrm{NH}), 1.84\left(\mathrm{~s}, 12 \mathrm{H}, \mathrm{CH}_{3}\right), 2.63\left(\mathrm{~s}, 6 \mathrm{H}, \mathrm{CH}_{3}\right), 3.03\left(\mathrm{~m}, 4 \mathrm{H}, \mathrm{CH}_{2} \mathrm{P}=\mathrm{S}\right), 3.42\left(\mathrm{~m}, 4 \mathrm{H}, \mathrm{SCH}_{2}\right), 7.31$ (s, 4H, mesityl $H$ ), $7.55(\mathrm{~m}, 16 \mathrm{H}, \mathrm{Ar} H), 7.89(\mathrm{~m}, 8 \mathrm{H}, \mathrm{Ar} H), 8.13\left(\mathrm{~d}, 4 \mathrm{H}, J_{\mathrm{H}-\mathrm{H}}=8.1 \mathrm{~Hz}\right.$, $\mathrm{ArH}), 8.69\left(\mathrm{~d}, 4 \mathrm{H}, J_{\mathrm{H}-\mathrm{H}}=5.1 \mathrm{~Hz}, \mathrm{Ar} H\right), 8.82\left(\mathrm{~d}, 4 \mathrm{H}, J_{\mathrm{H}-\mathrm{H}}=4.8 \mathrm{~Hz}, \mathrm{Ar} H\right) .{ }^{31} \mathrm{P}\left\{{ }^{1} \mathrm{H}\right\} \mathrm{NMR}$ $\left(\mathrm{CD}_{2} \mathrm{Cl}_{2}\right): \delta 41.6(\mathrm{~s})$. ESIMS $(\mathrm{m} / \mathrm{z})$ : Calcd. $1251.6\left[\mathrm{M}^{+}\right]$. Found: 1251.3. Elemental analysis for $\mathrm{C}_{78} \mathrm{H}_{68} \mathrm{~N}_{4} \mathrm{P}_{2} \mathrm{~S}_{4}$ : Calcd. C, 74.85; H, 5.48; N, 4.48. Found: C, 74.35; H, 4.98; N, 4.43. 


\section{[5,15-Bis-[4-(2-diphenylphosphinothioylethylsulfanyl)phenyl]-10,-20-bis(mesityl) porphyrinato] zinc(II) (12)}

In a $250-\mathrm{mL}$ round-bottom flask, $11(400 \mathrm{mg}, 0.319 \mathrm{mmol})$ and $\mathrm{Zn}(\mathrm{OAc})_{2} \cdot 2 \mathrm{H}_{2} \mathrm{O}(700 \mathrm{mg}$, $3.19 \mathrm{mmol})$ were refluxed for $3 \mathrm{~h}$ in a $\mathrm{CHCl}_{3} / \mathrm{CH}_{3} \mathrm{OH}(4: 1 \mathrm{v} / \mathrm{v}, 200 \mathrm{~mL})$ solution. The solution was washed with $\mathrm{H}_{2} \mathrm{O}(100 \mathrm{~mL})$ and extracted with $\mathrm{CHCl}_{3}(2 \times 50 \mathrm{~mL})$. The organic layer was washed again with $\mathrm{H}_{2} \mathrm{O}(50 \mathrm{~mL})$, dried over $\mathrm{Na}_{2} \mathrm{SO}_{4}$, and concentrated to give 12 as a purple microcrystalline solid (412 mg, 98\% yield). ${ }^{1} \mathrm{H}$ NMR $\left(\mathrm{CD}_{2}-\mathrm{Cl}_{2}\right): \delta 1.82\left(\mathrm{~s}, 12 \mathrm{H}, \mathrm{CH}_{3}\right), 2.63$ (s, $\left.6 \mathrm{H}, \mathrm{CH}_{3}\right), 3.03\left(\mathrm{~m}, 4 \mathrm{H}, \mathrm{CH}_{2} \mathrm{P}=\mathrm{S}\right), 3.41\left(\mathrm{~m}, 4 \mathrm{H}, \mathrm{SCH}_{2}\right), 7.31(\mathrm{~s}, 4 \mathrm{H}, \mathrm{ArH}), 7.54(\mathrm{~m}, 12 \mathrm{H}$, $\operatorname{ArH}), 7.89(\mathrm{~m}, 12 \mathrm{H}, \mathrm{Ar} H), 8.12\left(\mathrm{~d}, 4 \mathrm{H}, J_{\mathrm{H}-\mathrm{H}}=8.4 \mathrm{~Hz}, \operatorname{Ar} H\right), 8.76\left(\mathrm{~d}, 4 \mathrm{H}, J_{\mathrm{H}-\mathrm{H}}=5.1 \mathrm{~Hz}\right.$, $\mathrm{Ar} H), 8.89\left(\mathrm{~d}, 4 \mathrm{H}, J_{\mathrm{H}-\mathrm{H}}=4.8 \mathrm{~Hz}, \operatorname{Ar} H\right) .{ }^{31} \mathrm{P}\left\{{ }^{1} \mathrm{H}\right\} \operatorname{NMR}\left(\mathrm{CD}_{2}-\mathrm{Cl}_{2}\right): \delta 41.6(\mathrm{~s})$. ESIMS $(\mathrm{m} / \mathrm{z})$ : Calcd. 1314.9 $\left[\mathrm{M}^{+}\right]$. Found: 1314.1. Elemental analysis for $\mathrm{C}_{78} \mathrm{H}_{66} \mathrm{~N}_{4} \mathrm{P}_{2} \mathrm{~S}_{4} \mathrm{Zn}$ : Calcd. C, 71.24; H, 5.06; N, 4.26. Found: C, 70.69; H, 4.97; N, 3.94.

\section{[5,15-Bis-[4-(2-diphenylphosphanylethylsulfanyl)phenyl]-10,20-bis(mesityl)porphyrinato] zinc(II) (13)}

In a 50-mL Schlenk flask, 12 (300 mg, $0.228 \mathrm{mmol})$ and $\mathrm{Cp}_{2} \mathrm{ZrHCl}(382 \mathrm{mg}, 1.48 \mathrm{mmol})$ were stirred in THF $(40 \mathrm{~mL})$ at $60^{\circ} \mathrm{C}$ for $3 \mathrm{~h}$. The solvent was removed, and the product was purified via column chromatography (THF, silica gel) in a glove box under an atmosphere of $\mathrm{N}_{2}$. The solvent was removed in vacuo to yield $\mathbf{1 3}$ as a purple microcrystalline solid $(251 \mathrm{mg}, 88 \%$

yield). ${ }^{1} \mathrm{H}$ NMR (THF- $\left.d_{8}\right): \delta 1.84\left(\mathrm{~s}, 12 \mathrm{H}, \mathrm{CH}_{3}\right), 2.47\left(\mathrm{~m}, 4 \mathrm{H}, \mathrm{CH}_{2} \mathrm{P}\right), 2.61\left(\mathrm{~s}, 6 \mathrm{H}, \mathrm{CH}_{3}\right), 3.23$ $\left(\mathrm{m}, 4 \mathrm{H}, \mathrm{SCH}_{2}\right), 7.29(\mathrm{br} \mathrm{m}, 28 \mathrm{H}, \mathrm{Ar} H), 8.09\left(\mathrm{~d}, 4 \mathrm{H}, J_{\mathrm{H}-\mathrm{H}}=7.8 \mathrm{~Hz}, \mathrm{Ar} H\right), 8.64\left(\mathrm{~d}, 4 \mathrm{H}, J_{\mathrm{H}-\mathrm{H}}\right.$ $=4.8 \mathrm{~Hz}, \operatorname{Ar} H), 8.79\left(\mathrm{~d}, 4 \mathrm{H}, J_{\mathrm{H}-\mathrm{H}}=4.2 \mathrm{~Hz}, \operatorname{Ar} H\right) .{ }^{31} \mathrm{P}\left\{{ }^{1} \mathrm{H}\right\} \mathrm{NMR}\left(\mathrm{THF}-d_{8}\right): \delta-15.2(\mathrm{~s})$. ESIMS $(\mathrm{m} / \mathrm{z})$ : Calcd. $1250.8\left[\mathrm{M}^{+}\right]$. Found: 1249.4. Elemental analysis for $\mathrm{C}_{78} \mathrm{H}_{66} \mathrm{~N}_{4} \mathrm{P}_{2} \mathrm{~S}_{2} \mathrm{Zn}$ : Calcd. C, 74.90; H, 5.32; N, 4.48. Found: C, 73.15; H, 4.94; N, 4.16.

\section{[(13)Rh $\left.\left(\mathrm{BF}_{4}\right)\right]_{2}$ Condensed Macrocycle (14a)}

A small vial was charged with $[\mathrm{Rh}(\mathrm{NBD}) \mathrm{Cl}]_{2}(31.0 \mathrm{mg}, 0.068 \mathrm{mmol}), \mathrm{AgBF}_{4}(26.0 \mathrm{mg}, 0.135$ $\mathrm{mmol}$ ), and $\mathrm{CH}_{2} \mathrm{Cl}_{2}(4 \mathrm{~mL})$. This solution was stirred for $1 \mathrm{~h}$ and then filtered dropwise through Celite into a Schlenk flask. The red solution was then diluted with $\mathrm{CH}_{2} \mathrm{Cl}_{2}(20 \mathrm{~mL})$ to give a clear yellow/orange solution. A solution of ligand $\mathbf{1 3}$ (170 mg, $0.135 \mathrm{mmol})$ in THF (20 mL) was added to the "Rh" solution dropwise over $5 \mathrm{~min}$ at room temperature to give a dark-purple solution, which was then stirred for an additional $3 \mathrm{~h}$. The solvent was removed to yield $\mathbf{1 4 a}$ as a purple microcrystalline solid which was recrystallized from $\mathrm{CH}_{2} \mathrm{Cl}_{2} /$ pentane $(176 \mathrm{mg}$, 90\% yield). ${ }^{1} \mathrm{H}$ NMR $\left(\mathrm{CD}_{2} \mathrm{Cl}_{2}\right): \delta 1.48$ (br s, $\left.24 \mathrm{H}, \mathrm{CH}_{3}\right), 2.44$ (br s, $\left.12 \mathrm{H}, \mathrm{CH}_{3}\right), 2.76$ (br m, $8 \mathrm{H}, \mathrm{CH}_{2} \mathrm{P}$ ), 3.20 (br m, 8H, CH $\mathrm{CH}_{2} \mathrm{~S}$ ), 6.98 (br m, 8H, $\mathrm{ArH}$ ), $7.37-7.59$ (br m, 40H, $\mathrm{ArH}$ ), 8.02 (br m, $16 \mathrm{H}, \mathrm{Ar} H$ ), 8.50 (br m, $16 \mathrm{H}, \mathrm{Ar} H) .{ }^{31} \mathrm{P}\left\{{ }^{1} \mathrm{H}\right\} \mathrm{NMR}\left(\mathrm{CD}_{2} \mathrm{Cl}_{2}\right): \delta 64.1\left(\mathrm{~d}, J_{\mathrm{Rh}-\mathrm{P}}=162\right.$ $\mathrm{Hz})$. ESIMS (m/z) for $\left[\mathrm{C}_{156} \mathrm{H}_{132} \mathrm{~N}_{8} \mathrm{~S}_{4} \mathrm{P}_{4} \mathrm{Rh}_{2} \mathrm{Zn}_{2}\right]^{2+}$ : Calcd.1353.7. Found: 1354.1. Elementalanalysisfor $\left[\mathrm{C}_{156} \mathrm{H}_{132} \mathrm{~B}_{2} \mathrm{~F}_{8} \mathrm{~N}_{8} \mathrm{~S}_{4} \mathrm{P}_{4}-\mathrm{Rh}_{2} \mathrm{Zn}_{2}\right]$ : Calcd. C, 65.03; H, 4.62; N, 3.89 . Found: C, 65.56; H, 4.11; N, 3.46 .

\section{$\left[(13) \mathrm{Cu}\left(\mathrm{PF}_{6}\right)\right]_{2}$ Condensed Macrocycle (14b)}

A 50-mL Schlenk flask was charged with $\left[\mathrm{Cu}\left(\mathrm{CH}_{3} \mathrm{CN}\right)_{4}\right] \mathrm{PF}_{6}(29.8 \mathrm{~g}, 0.0799 \mathrm{mmol})$. The $\mathrm{Cu}^{\mathrm{I}}$ precursor was dissolved in $\mathrm{CH}_{2} \mathrm{Cl}_{2}(5 \mathrm{~mL})$, and a THF solution of ligand $\mathbf{1 3}$ (100 mg, 0.0799 mmol, $20 \mathrm{~mL}$ ) was added to the "Cu" solution dropwise over $5 \mathrm{~min}$ at room temperature to give a red/purple solution. The solution was then allowed to stir for $3 \mathrm{~h}$. The solvent was removed to yield $\mathbf{1 4 b}$ as a purple microcrystalline solid which was recrystallized from $\mathrm{CH}_{2} \mathrm{Cl}_{2} /$ pentane (105 mg, 90\% yield). ${ }^{1} \mathrm{H}$ NMR $\left(\mathrm{CD}_{2} \mathrm{Cl}_{2}\right): \delta 1.75$ (br s, $\left.24 \mathrm{H}, \mathrm{CH}_{3}\right), 2.55$ (br s, $12 \mathrm{H}, \mathrm{CH}_{3}$ ), $3.00\left(\right.$ br m, $8 \mathrm{H}, \mathrm{CH}_{2} \mathrm{P}$ ), 3.65 (br m, 8H, $\mathrm{CH}_{2} \mathrm{~S}$ ), $7.24-7.82$ (br m, $56 \mathrm{H}, \mathrm{ArH}$ ), 8.16 (br m, 8H, $\mathrm{Ar} H), 8.76$ (br s, $16 \mathrm{H}, \mathrm{Ar} H) .{ }^{31} \mathrm{P}\left\{{ }^{1} \mathrm{H}\right\} \mathrm{NMR}\left(\mathrm{CD}_{2} \mathrm{Cl}_{2}\right): \delta 1.0(\mathrm{~s})$. ESIMS $(\mathrm{m} /$ z) for $\left[\mathrm{C}_{156} \mathrm{H}_{132} \mathrm{~N}_{8} \mathrm{~S}_{4} \mathrm{P}_{4} \mathrm{Cu}_{2} \mathrm{Zn}_{2}\right]^{2+}$ : Calcd. 1314.4. Found: 1314.5. Elemental analysis for 
$\left[\mathrm{C}_{156} \mathrm{H}_{132} \mathrm{~N}_{8} \mathrm{~S}_{4} \mathrm{P}_{6} \mathrm{~F}_{12} \mathrm{Cu}_{2} \mathrm{Zn}_{2}\right] \mathrm{CH}_{2}-\mathrm{Cl}_{2}$ : Calcd. C, 62.78; H, 4.50; N, 3.73. Found: C, 61.95; H, $4.18 ; \mathrm{N}, 3.31$.

\section{[(13)RhCl(CO) $]_{2}$ Macrocycle (15a)}

Compound 14a (20.0 mg, $0.00694 \mathrm{mmol}$ ) was dissolved in $\mathrm{CD}_{2} \mathrm{Cl}_{2}(1 \mathrm{~g})$ and placed in an airfree NMR tube. PPNCl (Bis(triphenylphosphoranylidene) ammonium chloride, 2 equiv) was added and the NMR tube was then pressurized with $\mathrm{CO}(1 \mathrm{~atm})$ for $30 \mathrm{~s}$. Compound 14a was quantitatively converted to $15 \mathbf{a}$ as determined by ${ }^{31} \mathrm{P}$ NMR spectroscopy. ${ }^{1} \mathrm{H}$ NMR $\left(\mathrm{CD}_{2} \mathrm{Cl}_{2}\right)$ : $\delta 1.77$ (br s, 24H, $\mathrm{CH}_{3}$ ), 2.54 (br s, $12 \mathrm{H}, \mathrm{CH}_{3}$ ), 3.14 (br m, $8 \mathrm{H}, \mathrm{CH}_{2} \mathrm{P}$ ), 3.48 (br m, $8 \mathrm{H}$, $\left.\mathrm{CH}_{2} \mathrm{~S}\right), 7.26-8.01$ (br m, $\left.124 \mathrm{H},\left(\mathrm{C}_{6} \mathrm{H}_{5}\right)_{3} \mathrm{PPN}, \mathrm{P}\left(\mathrm{C}_{6} \mathrm{H}_{5}\right)_{2}, \mathrm{ArH}\right), 8.65-8.80$ (br m, $16 \mathrm{H}$, $\mathrm{Ar} H) .{ }^{31} \mathrm{P}\left\{{ }^{1} \mathrm{H}\right\} \mathrm{NMR}\left(\mathrm{CD}_{2} \mathrm{Cl}_{2}\right): \delta 22.1(\mathrm{~s}, \mathrm{PPNCl}), \delta 25.1\left(\mathrm{~d}, J_{\mathrm{Rh}-\mathrm{P}}=123 \mathrm{~Hz}\right)$. ESIMS $(\mathrm{m} / z)$ for $\left[\mathrm{C}_{156} \mathrm{H}_{132} \mathrm{~N}_{8} \mathrm{~S}_{4} \mathrm{P}_{4}-\mathrm{Rh}_{2} \mathrm{Zn}_{2}\right]^{2+}\left(\mathrm{M}-2\left(\mathrm{Cl}^{-} / \mathrm{CO}\right)\right)$ : Calcd. 1353.7. Found: 1353.7.

\section{[(13) Cu(pyridine- $\left.\left.d_{5}\right)_{2}\left(\mathrm{PF}_{6}\right)\right]_{2}$ Macrocycle (15b)}

Compound $14 \mathbf{b}(20.0 \mathrm{mg}, 0.00640 \mathrm{mmol})$ was dissolved in $\mathrm{CD}_{2} \mathrm{Cl}_{2}(1 \mathrm{~g})$ and placed in an airfree NMR tube. $\mathrm{C}_{5} \mathrm{D}_{5} \mathrm{~N}$ (4 equiv.) was added to this solution and the conversion of $\mathbf{1 5 b}$ was quantitative as determined by ${ }^{1} \mathrm{H}$ NMR and ${ }^{31} \mathrm{P}$ NMR spectroscopy. ${ }^{1} \mathrm{H}$ NMR $\left(\mathrm{CD}_{2} \mathrm{Cl}_{2}\right): \delta 1.72$ (br s, $24 \mathrm{H}, \mathrm{CH}_{3}$ ), 2.45 (br s, 12H, $\mathrm{CH}_{3}$ ), 2.82 (br m, $8 \mathrm{H}, \mathrm{CH}_{2} \mathrm{P}$ ), 3.38 (br m, $8 \mathrm{H}, \mathrm{CH}_{2} \mathrm{~S}$ ), 7.17 $-7.55(\mathrm{br} \mathrm{m}, 56 \mathrm{H}, \mathrm{ArH}), 8.04\left(\mathrm{~d}, 8 \mathrm{H}, J_{\mathrm{H}-\mathrm{H}}=7.8 \mathrm{~Hz}, \mathrm{Ar} H\right), 8.64\left(\mathrm{~d}, 8 \mathrm{H}, J_{\mathrm{H}-\mathrm{H}}=4.5 \mathrm{~Hz}\right.$, $\operatorname{Ar} H), 8.70\left(\mathrm{~d}, 8 \mathrm{H}, J_{\mathrm{H}-\mathrm{H}}=4.8 \mathrm{~Hz}, \operatorname{Ar} H\right) .{ }^{31} \mathrm{P}\left\{{ }^{1} \mathrm{H}\right\} \mathrm{NMR}\left(\mathrm{CD}_{2} \mathrm{Cl}_{2}\right): \delta-8.0(\mathrm{~s})$. ESIMS $(\mathrm{m} / z)$ for $\left[\mathrm{C}_{156} \mathrm{H}_{132} \mathrm{~N}_{8} \mathrm{~S}_{4} \mathrm{P}_{4} \mathrm{Cu}_{2} \mathrm{Zn}_{2}\right]^{2+}$ : Calcd. 1314.4. Found: 1314.9.

\section{[( $\left.\left.\mathrm{Ph}_{2} \mathrm{PCH}_{2} \mathrm{CH}_{2} \mathrm{~S}-\mathrm{C}_{6} \mathrm{H}_{2}\left(\mathrm{CH}_{3}\right)_{3}\right)_{2} \mathrm{Rh}\right] \mathrm{BF}{ }_{4}$ Condensed Tweezer-Type Complex (16a)}

A $20-\mathrm{mL}$ vial was charged with $[\mathrm{Rh}(\mathrm{NBD}) \mathrm{Cl}]_{2}(63.2 \mathrm{mg}, 0.137 \mathrm{mmol}), \mathrm{AgBF}_{4}(53.4 \mathrm{mg}, 0.274$ $\mathrm{mmol})$, and $\mathrm{CH}_{2} \mathrm{Cl}_{2}(4 \mathrm{~mL})$. This solution was stirred for $1 \mathrm{~h}$ and then filtered dropwise through Celite into a Schlenk flask. The orange solution was then diluted with $\mathrm{CH}_{2} \mathrm{Cl}_{2}(20 \mathrm{~mL})$ to give a clear yellow solution. A solution of (2-(mesitylthio)ethyl)diphenylphosphine ( $200 \mathrm{mg}, 0.549$ $\mathrm{mmol})$ in $\mathrm{CH}_{2}-\mathrm{Cl}_{2}(20 \mathrm{~mL})$ was added to the " $\mathrm{Rh}$ " solution dropwise over $5 \mathrm{~min}$ at room temperature to give a clear yellow solution, which was then stirred for an additional $3 \mathrm{~h}$. The solvent was removed to yield $\mathbf{1 6 a}$ as a yellow microcrystalline solid which was recrystallized from $\mathrm{CH}_{2} \mathrm{Cl}_{2} /$ pentane (113 mg, 90\% yield). ${ }^{1} \mathrm{H} \mathrm{NMR}\left(\mathrm{CD}_{2} \mathrm{Cl}_{2}\right): \delta 2.22\left(\mathrm{~d}, 12 \mathrm{H}, \mathrm{CH}_{3}\right), 2.51$ (br s, $10 \mathrm{H}, \mathrm{CH}_{3}$ and $\mathrm{SCH}_{2} \mathrm{CH}_{2} \mathrm{P}$ ), 2.83 (br m, 4H, $\mathrm{CH}_{2} \mathrm{P}$ ), 6.71 (s, $\left.4 \mathrm{H}, \mathrm{ArH}\right), 7.23-7.34$ (br $\left.\mathrm{m}, \mathrm{P}(\mathrm{Ar}-\mathrm{H})_{2}, 20 \mathrm{H}\right) .{ }^{31} \mathrm{P}\left\{{ }^{1} \mathrm{H}\right\}$ NMR $\left(\mathrm{CD}_{2} \mathrm{Cl}_{2}\right): \delta 63.0\left(\mathrm{~d}, J_{\mathrm{Rh}}-\mathrm{P}=163 \mathrm{~Hz}\right)$. ESIMS $(\mathrm{m} / z)$ for $\left[\mathrm{C}_{46} \mathrm{H}_{50} \mathrm{~N}_{8} \mathrm{~S}_{2} \mathrm{P}_{2} \mathrm{Rh}\right]^{+}$: Calcd. 831.8. Found: 831.6.

\section{$\left(\mathrm{Ph}_{2} \mathrm{PCH}_{2} \mathrm{CH}_{2} \mathrm{~S}-\mathrm{C}_{6} \mathrm{H}_{2}\left(\mathrm{CH}_{3}\right)_{3}\right)_{2} \mathrm{RhCl}(\mathrm{CO})$ Open Tweezer-Type Complex (16b)}

Compound 16a (15.0 mg, $0.0163 \mathrm{mmol})$ was dissolved in $\mathrm{CD}_{2} \mathrm{Cl}_{2}(1 \mathrm{~g})$ and placed in an airfree NMR tube. PPNCl (bis(triphenylphosphoranylidene)ammonium chloride, $9.38 \mathrm{mg}$, $0.0163 \mathrm{mmol}$ ) was added as a solid and the NMR tube was then pressurized with $\mathrm{CO}$ (1 atm) for $30 \mathrm{~s}$. Compound 16a was quantitatively converted to $\mathbf{1 6} \mathbf{b}$ as determined by ${ }^{31} \mathrm{P}\left\{{ }^{1} \mathrm{H}\right\}$ NMR spectroscopy. ${ }^{1} \mathrm{H}$ NMR $\left(\mathrm{CD}_{2}-\mathrm{Cl}_{2}\right): \delta 2.25\left(\mathrm{~s}, 6 \mathrm{H}, \mathrm{CH}_{3}\right), 2.37\left(\mathrm{~s}, 12 \mathrm{H}, \mathrm{CH}_{3}\right), 2.67$ (br m, 4H, $\left.\mathrm{CH}_{2} \mathrm{P}\right), 2.82$ (br m, 4H, CH $\left.\mathrm{CH}_{2} \mathrm{~S}\right), 6.90$ (s, $\left.4 \mathrm{H}, \mathrm{ArH}\right), 7.34-7.65$ (br m, 50H, $\left(\mathrm{C}_{6} \mathrm{H}_{5}\right)_{3} \mathrm{PPN}, \mathrm{P}(\mathrm{Ar}-$ $\left.H)_{2}\right) .{ }^{31} \mathrm{P}\left\{{ }^{1} \mathrm{H}\right\} \mathrm{NMR}\left(\mathrm{CD}_{2} \mathrm{Cl}_{2}\right): \delta 22.1(\mathrm{~s}, \mathrm{PPNCl}), \delta 23.5\left(\mathrm{~d}, J_{\mathrm{Rh}-\mathrm{P}}=123 \mathrm{~Hz}\right)$. ESIMS $(\mathrm{m} / \mathrm{z})$ for $\left[\mathrm{C}_{46} \mathrm{H}_{50} \mathrm{~N}_{8} \mathrm{~S}_{2} \mathrm{P}_{2} \mathrm{Rh}\right]^{+}\left(\mathrm{M}-\left(\mathrm{Cl}^{-} / \mathrm{CO}\right)\right)$ : Calcd. 831.8. Found: 831.2.

\section{General Procedure for Catalysis Experiments}

The formation of 2-, 3-, and 4-acetoxymethylpyridine were monitored by GC relative to an internal standard (biphenyl) and quantified using a previously established calibration curve. Experiments containing 14a, 15a, or a mixture of an analogous monomeric $\mathrm{Rh}^{\mathrm{I}}$ complex (16a or 16b) and $\mathbf{Z n ( T P P ) ~ ( a s ~ t h e ~ c o n t r o l ) , ~ r e s p e c t i v e l y , ~ w e r e ~ r u n ~ c o n c u r r e n t l y ~ i n ~ s e p a r a t e ~}$ vials at room temperature in $\mathrm{CH}_{2} \mathrm{Cl}_{2}$ under an atmosphere of $\mathrm{N}_{2}$ inside a glove box. 
GC Experiments for the Closed Macrocycle (14a)-Inside a glove box, $\mathrm{CH}_{2} \mathrm{Cl}_{2}$ stock solutions of complex 14a $(0.58 \mathrm{~mL}$ of a $2.6 \mathrm{mM}$ solution), biphenyl $(0.5 \mathrm{~mL}$ of a $25 \mathrm{mM}$ solution), and pyridylcarbinol ( $0.5 \mathrm{~mL}$ of a $90 \mathrm{mM}$ solution) were added to a $20-\mathrm{mL}$ vial. Fresh $\mathrm{CH}_{2}-\mathrm{Cl}_{2}$ was added to the vial bringing the total reaction volume up to $4.5 \mathrm{~mL}$. After stirring for $5 \mathrm{~min}, 1$-acetylimidazole $(0.5 \mathrm{~mL}$ of a $60 \mathrm{mM}$ solution) was added to the vial $(t=0)$. At various times, an aliquot $(100 \mu \mathrm{L})$ was taken from the solution and added to diethyl ether (2 $\mathrm{mL})$. This was then passed down a plug of Celite $(3 \mathrm{~cm} \times 0.5 \mathrm{~cm})$ to remove the catalyst. The plug was further treated with fresh diethyl ether $(5 \mathrm{~mL})$. The combined organics were used for GC analysis.

GC Experiments for the Open Macrocycle (15a)-Inside a glove box, complex 14a $(0.58 \mathrm{~mL}$ of a $2.6 \mathrm{mM}$ solution) and benzyltriethylammonium chloride $(0.5 \mathrm{~mL}$ of a $6.4 \mathrm{mM}$ solution) were added to a 10-mL Schlenk flask. The flask was removed from the glove box and placed on a Schlenk line where CO (1 atm) was bubbled through the solution for $30 \mathrm{~s}$ resulting in the formation of 15a. The flask was brought back into the glove box and the contents were transferred into a vial. Fresh $\mathrm{CH}_{2} \mathrm{Cl}_{2}$ was added to the flask and transferred into the vial bringing the total reaction volume up to approximately $4 \mathrm{~mL}$. Pyridylcarbinol $(0.5 \mathrm{~mL}$ of a 90 $\mathrm{mM}$ solution) and biphenyl ( $0.5 \mathrm{~mL}$ of a $25 \mathrm{mM}$ solution) were added to the vial and after stirring for $5 \mathrm{~min}, 1$-acetylimidazole $(0.5 \mathrm{~mL}$ of a $60 \mathrm{mM}$ solution) was added to the flask $(t=$ $0)$. At various times, an aliquot $(100 \mu \mathrm{L})$ was taken from the solution and added to diethyl ether $(2 \mathrm{~mL})$. This was then passed down a plug of Celite $(3 \mathrm{~cm} \times 0.5 \mathrm{~cm})$ to remove the catalyst. The plug was further treated with fresh diethyl ether $(5 \mathrm{~mL})$. The resulting samples were used for GC analysis.

\section{GC Control Experiments for $\mathbf{R h}^{\mathbf{l}}$-Monomer (16a) and Porphyrin Monomer (Zn}

(TPP))-Inside a glove box, $\mathrm{CH}_{2} \mathrm{Cl}_{2}$ stock solutions of complex $16 \mathbf{a}(0.892 \mathrm{~mL}$ of a $8.0 \mathrm{mM}$ solution), biphenyl ( $0.5 \mathrm{~mL}$ of a $25 \mathrm{mM}$ solution), $\mathrm{Zn}$ (TPP) $(0.20 \mathrm{~mL}$ of a $15 \mathrm{mM}$ solution), and pyridylcarbinol $(0.5 \mathrm{~mL}$ of a $90 \mathrm{mM}$ solution) were added to a $20-\mathrm{mL}$ vial. Fresh $\mathrm{CH}_{2} \mathrm{Cl}_{2}$ was added to the vial bringing the total reaction volume up to $4.5 \mathrm{~mL}$. After stirring for $5 \mathrm{~min}, 1$-acetylimidazole $(0.5 \mathrm{~mL}$ of a $60 \mathrm{mM}$ solution) was added to the vial $(t=0)$. At various times, an aliquot $(100 \mu \mathrm{L})$ was taken from the solution and added to diethyl ether (2 $\mathrm{mL})$. This was then passed down a plug of Celite $(3 \mathrm{~cm} \times 0.5 \mathrm{~cm})$ to remove the catalyst. The plug was further treated with fresh diethyl ether $(5 \mathrm{~mL})$. The combined organics were used for GC analysis.

GC Control Experiments for Rh'-Monomer (16b) and Porphyrin Monomer-Inside a glove box, complex $16 a(0.892 \mathrm{~mL}$ of a $8.0 \mathrm{mM}$ solution), $\mathrm{Zn}$ (TPP) $(0.20 \mathrm{~mL}$ of a $15 \mathrm{mM}$ solution), and benzyltriethylammonium chloride $(1.0 \mathrm{~mL}$ of a $6.4 \mathrm{mM}$ solution) were added to a $10-\mathrm{mL}$ Schlenk flask. The flask was removed from the glove box and placed on a Schlenk line where $\mathrm{CO}(1 \mathrm{~atm})$ was bubbled through the solution for $30 \mathrm{~s}$ resulting in the formation of 16b. The flask was brought back into the glove box and the contents were transferred into a vial. Fresh $\mathrm{CH}_{2} \mathrm{Cl}_{2}$ was added to the flask and transferred into the vial bringing the total reaction volume up to approximately $4 \mathrm{~mL}$. Pyridylcarbinol $(0.5 \mathrm{~mL}$ of a $90 \mathrm{mM}$ solution) and biphenyl $(0.5 \mathrm{~mL}$ of a $25 \mathrm{mM}$ solution) were added to the vial and after stirring for $5 \mathrm{~min}, 1-$ acetylimidazole $(0.5 \mathrm{~mL}$ of a $60 \mathrm{mM}$ solution $)$ was added to the flask $(t=0)$. At various times, an aliquot $(100 \mu \mathrm{L})$ was taken from the solution and added to diethyl ether $(2 \mathrm{~mL})$. This was then passed down a plug of Celite $(3 \mathrm{~cm} \times 0.5 \mathrm{~cm})$ to remove the catalyst. The plug was further treated with fresh diethyl ether $(5 \mathrm{~mL})$. The combined organics were used for $\mathrm{GC}$ analysis. 


\section{Results and Discussion}

\section{The Design of Ether-Phosphine Hemilabile Ligand 7}

To design porphyrin-based supramolecular systems capable of in situ allosteric activity, flexibility must be engineered into the backbone of the framework as well as "weak" and "strong" binding structural domains. To this end, we synthesized two new hemilabile porphyrin ligands which incorporate "weak" ether or thioether functionalities in addition to "strong" phosphine binding sites. Ether-based ligand 7 was obtained in eight steps from 4hydroxybenzaldehyde (Scheme 2). 4-Hydroxy-benzaldehyde was alkylated with excess 1bromo-2-chloroethane to yield 4-(2-chloroethoxy)benzaldehyde, which was transformed into 3 after a dithiane protection of the aldehyde, conversion of the chloride to the corresponding phosphine, and reaction with elemental sulfur. Deprotection of the dithiane moiety in $\mathbf{3}$, followed by condensation of the resulting aldehyde with 5-mesityldipyrromethane affords the freebase porphyrin 5 in gram quantities after column chromatography. Metalation with $\mathrm{Zn}$ $(\mathrm{OAc})_{2} \cdot 2 \mathrm{H}_{2} \mathrm{O}$ gave compound $\mathbf{6}$, and desulfurization with Schwartz's reagent gave ligand 7 in $12 \%$ overall yield. The use of a sulfide as the protecting group for the phosphine moiety not only allowed for a convenient nonaqueous protection/deprotection sequence, but also significantly increased the scalability and the yield of the porphyrin synthesis while simplifying the complicated purification and isolation process that often accompanies porphyrin syntheses. Indeed, if one uses an oxide protecting group as opposed to the sulfide, the ligand adheres to the silica column used for chromatography and makes the isolation of the pure product difficult.

\section{Reactivity of Ligand 7 with $\mathrm{Rh}^{\mathrm{l}}$ and $\mathrm{Cu} \mathrm{I}^{\mathrm{I}}$}

Initially, our attention was focused on the design, synthesis, and isolation of $\mathrm{Rh}^{\mathrm{I}}$ and $\mathrm{Cu}^{\mathrm{I}}$ condensed intermediates of type I using ligand 7. Unfortunately, isolation of these products proved impossible under the conditions explored. However, we discovered that the "open" macrocyclic structure 8a can be obtained directly in quantitative yield upon reaction of ligand 7 with $\left[\mathrm{Rh}(\mathrm{CO})_{2}(\mathrm{Cl})\right]_{2}$. The ${ }^{31} \mathrm{P}\left\{{ }^{1} \mathrm{H}\right\}$ NMR spectrum of $8 \mathbf{a}$ exhibits a single resonance at $\delta$ $21.6\left(\mathrm{~d}, J_{\mathrm{Rh}-\mathrm{P}}=124 \mathrm{~Hz}\right)$, diagnostic of a highly symmetrical porphyrin complex with transphosphines and is consistent with the proposed structural formulation for $\mathbf{8 a} .{ }^{50}$ ESIMS analysis of 8a shows a parent ion at $m / z$ 1349.3, indicating the loss of each $\mathrm{Cl}^{-}$ligand bound to the $\mathrm{Rh}^{\mathrm{I}}$ centers. $\mathrm{Rh}^{\mathrm{I}}$ complexes with analogous coordination environments often lose these ligands during ESIMS. 50

Similar to the reactivity exhibited with $\mathrm{Rh}^{\mathrm{I}}$, ligand $\mathbf{7}$ forms the analogous "open" macrocyclic product $8 \mathbf{b}$ when reacted with $\left[\mathrm{Cu}\left(\mathrm{CH}_{3} \mathrm{CN}\right)_{4}\right] \mathrm{PF}_{6}$. The ${ }^{31} \mathrm{P}\left\{{ }^{1} \mathrm{H}\right\}$ NMR spectrum of $\mathbf{8 b}$ exhibits a singlet at $\delta-11.5$ consistent with a tetrahedral $\mathrm{Cu}^{\mathrm{I}}-\mathrm{P}$ environment. ${ }^{51}$ The resonance is substantially shifted downfield from the one observed for free ligand at $\delta-21.2$. Characterization of this product via ESIMS leads to the observation of a parent ion at $\mathrm{m} / \mathrm{z}$ 1282.4 which corresponds to the loss of two $\mathrm{CH}_{3} \mathrm{CN}$ molecules from each $\mathrm{Cu}^{\mathrm{I}}$ metal center. This is consistent with the known lability of the bound $\mathrm{CH}_{3} \mathrm{CN}$ molecules, which has been observed in analogous systems. 51

\section{X-ray Structure Determination of $8 \mathrm{a} \subset \mathrm{DABCO}$ and $8 \mathrm{c} \subset \mathrm{DABCO}$}

Initial attempts to grow crystals of $\mathbf{8 a}$ in a variety of solvent mixtures proved unsuccessful, presumably due to the large free volume and flexibility of the cavity. We hypothesized that crystals of $8 \mathbf{a}$ could be obtained in the presence of a ligand that can span and coordinate to the two $\mathrm{Zn}$-centers within the macrocycle, filling the free volume and rigidifying the overall structure. Indeed, $\mathrm{X}$-ray quality crystals were obtained from vapor diffusion of pentane/diethyl ether $(1: 1 \mathrm{v} / \mathrm{v})$ into a THF/ $\mathrm{CH}_{2} \mathrm{Cl}_{2}(1: 1 \mathrm{v} / \mathrm{v})$ solution of $\mathbf{8 a}$ containing DABCO which was layered with acetonitrile. The 56-membered macrocyclic product $8 \mathbf{a} \subset \mathbf{D A B C O}$ contains two porphyrin moieties aligned and locked into a cofacial arrangement in the solid-state by a 
DABCO ligand (Figure 1). The $\mathrm{Zn}-\mathrm{Zn}$ distance of $7.09 \AA$ A is significantly longer than the P$\mathrm{Rh}-\mathrm{P}$ distance of 4.64 which is made possible by the flexible $\left(\mathrm{CH}_{2}\right)_{2} \mathrm{O}$ linkages between the porphyrin moieties and the $\mathrm{Rh}^{\mathrm{I}}$ hinges. This large porphyrin-porphyrin distance allows for the mesityl arms of the porphyrin moieties to align in a near superimposable fashion.

Attempts to crystallize $\mathbf{8 b}$ in a similar manner led to an unexpected reaction with the halogenated solvent. Because the solubility of $\mathbf{8 b}$ in pure THF is poor, a mixture of solvents proved to be the most effective for crystallization. Analytically pure samples of $\mathbf{8 b}$ were dissolved in a THF/ $\mathrm{CH}_{2} \mathrm{Cl}_{2}$ solution containing DABCO which was first layered with acetonitrile following by a slow second layer of a solution of pentane/diethyl ether $(1: 1 \mathrm{v} / \mathrm{v})$. $\mathrm{X}$-ray quality crystals were obtained from this mixture after 1 day and subjected to singlecrystal diffraction analysis at the Advanced Photon Source (APS, Argonne National Laboratory). Although $\mathbf{8 b}$ has been shown to contain four molecules of $\mathrm{CH}_{3} \mathrm{CN}$ and two $\mathrm{PF}_{6}{ }^{-}$counterions according to elemental analysis, the diffraction experiment yielded a structure with DABCO bridging both $\mathrm{Zn}$-atoms and two $\mathrm{Cl}^{-}$anions bound to each tetrahedral $\mathrm{Cu}$ metal center, indicating an oxidation of the original $\mathrm{Cu}^{\mathrm{I}}$ center to $\mathrm{Cu}^{\mathrm{II}}$ forming $8 \mathbf{C} \subset \mathbf{D A B C O}$ (see Supporting Information). Such a compound could be formed from the dehalogenation of alkyl halide solvents (i.e., $\mathrm{CH}_{2} \mathrm{Cl}_{2}$ and $\mathrm{CHCl}_{3}$ ) by the $\mathrm{Cu}^{\mathrm{I}}$ center in $\mathbf{8 b}$, similar to that observed by Karlin et al. for (TMPA)Cu ${ }^{\mathrm{I}}$ (TMPA= (tris-(2-pyridylmethyl)amine) ) in $\mathrm{CH}_{2} \mathrm{Cl}_{2} .{ }^{52-54}$ That $\mathbf{8 c} \subset \mathrm{DABCO}$ possesses the framework expected for $\mathbf{8 b}$ supports our assignment of an open macrocyclic structure for the product isolated from the reaction of 7 and $\left[\mathrm{Cu}\left(\mathrm{CH}_{3} \mathrm{CN}\right)_{4}\right] \mathrm{PF}_{6}$.

\section{Synthesis of Thioether-Phosphine Hemilabile Ligand 13}

Because type I structures are difficult to access from the ether-phosphine ligand 7, we hypothesized that isostructural ligands containing thioether linkages would be more effective at forming condensed structures as has been observed previously with analogous smaller molecules. 50 To this end, we modified the synthesis of 7 to obtain the thioether-based porphyrin ligand 13 in six steps (Scheme 3). First, 4-bromothiophenol was stoichiometrically alkylated with 1-chloro-2-diphenylphosphinoethane to yield 2-(4-bromophenylsulfanyl) ethyldiphenyl phosphine. To protect the phosphine moiety from oxidation during the porphyrin synthesis, reaction with elemental sulfur yields phosphine sulfide $\mathbf{9}$, which was then formylated with $n$-BuLi and DMF. The resulting aldehyde 10 was subsequently condensed with 5mesityldipyrromethane in the presence of $\mathrm{BF}_{3} \cdot \mathrm{OEt}_{2}$ to yield porphyrin 11 in $44 \%$ yield after a very simple chromatographic separation. Freebase porphyrin $\mathbf{1 1}$ was metallated with $\mathrm{Zn}$ $(\mathrm{OAc})_{2} \cdot 2 \mathrm{H}_{2} \mathrm{O}$ to give $\mathbf{1 2}$ and followed by deprotection of the phosphine with Schwartz's reagent to yield the phosphine derivative $\mathbf{1 3}$ in $33 \%$ overall yield.

\section{Reactivity of Hemilabile Ligand 13 with $\mathrm{Rh}^{\mathbf{l}}$ and $\mathrm{Cu}^{\mathbf{I}}$}

In contrast to the results obtained from ether ligand $\mathbf{7}$, thioether ligand $\mathbf{1 3}$ reacts cleanly with "Rh(NBD)BF 4 " 55 to form the condensed intermediate 14a. The ${ }^{31} \mathrm{P}\left\{{ }^{1} \mathrm{H}\right\}$ NMR spectrum of 14a exhibits a doublet at $\delta 64.5\left(J_{\mathrm{Rh}-\mathrm{P}}=162 \mathrm{~Hz}\right)$ which is highly diagnostic of its symmetrical structure and cis-Rh-P coordination centers. ${ }^{50}$ Additionally, ESIMS analysis shows a peak corresponding to the $\mathrm{M}^{2+}$ ion at $1354.1 \mathrm{~m} / \mathrm{z}$, indicating the formation of the desired condensed structure. Significantly, compound 14a can be opened into macrocycle 15a by introduction of benzyltriethyl ammonium chloride or by $\mathrm{PPNCl}$ (where $\mathrm{PPNCl}=$ bis

(triphenylphosphoranylidene)ammonium chloride) and $\mathrm{CO}$. This transformation can be followed by ${ }^{31} \mathrm{P}\left\{{ }^{1} \mathrm{H}\right\}$ NMR through the appearance of a doublet at $\delta 25.1\left(\mathrm{~d}, J_{\mathrm{P}-\mathrm{Rh}}=123 \mathrm{~Hz}\right)$, significantly upfield from the resonance for 14a. This resonance is characteristic of a transphosphine environment about the $\mathrm{Rh}^{\mathrm{I}}$ metal center and indicates a conversion from a condensed macrocyclic intermediate to the "open" macrocycle15a. 50 The ESIMS spectrum of 15a exhibits a peak at $1353.7 \mathrm{~m} / z$, corresponding to the $\mathrm{M}^{2+}$ ion without the $\mathrm{Cl}^{-}$and $\mathrm{CO}$ ligands and is consistent with ESIMS data for analogous structures. ${ }^{26}$ 
We observed condensed intermediate $\mathbf{1 4} \mathbf{b}$ as the sole product from the reaction between thioether-based ligand 13 and $\left[\mathrm{Cu}-\left(\mathrm{CH}_{3} \mathrm{CN}\right)_{4}\right] \mathrm{PF}_{6}$. The ${ }^{31} \mathrm{P}\left\{{ }^{1} \mathrm{H}\right\}$ NMR spectrum of $\mathbf{1 4 b}$ exhibits a singlet at $\delta 1.0$ which is highly diagnostic of a tetrahedral environment at the $\mathrm{Cu}^{\mathrm{I}}$ metal center. ${ }^{51}$ Additionally, ESIMS analysis yields a peak corresponding to the $\mathrm{M}^{2+}$ ion at $1314.5 \mathrm{~m} / \mathrm{z}$, indicating formation of the desired condensed structure. Notably, compound $\mathbf{1 4 b}$ can be converted into macrocycle $15 \mathbf{b}$ upon the addition of stoichiometric quantities of pyridine. Once again, the ${ }^{31} \mathrm{P}\left\{{ }^{1} \mathrm{H}\right\}$ NMR spectrum allows us to monitor this transformation through the appearance of a singlet at $\delta-8.0$, which has shifted upfield from the resonance observed for 14b. The ESIMS analysis of $\mathbf{1 5 b}$ yields a peak at $1314.9 \mathrm{~m} / \mathrm{z}$ which corresponds to the pyridine-free molecular ion.

\section{X-ray Structure Determination of $15 a \subset D A B C O$ and $15 c \subset D A B C O$}

Similar to the problems encountered in crystallizing $\mathbf{8 a}$, our attempts to isolate X-ray quality single crystals of 14a and 15a proved unfruitful. Based upon the more promising strategy of adding DABCO to pure samples of $\mathbf{8 a}$, we hypothesized that crystals of $\mathbf{1 5 a} \subset \mathbf{D A B C O}$ could be obtained in a similar fashion. X-ray quality crystals were obtained from vapor diffusion of pentane/diethyl ether $(1: 1 \mathrm{v} / \mathrm{v})$ into a THF/ $\mathrm{CH}_{2} \mathrm{Cl}_{2}$ solution of $\mathbf{1 5 a}$ containing DABCO, which was initially layered with acetonitrile. Similar to the analogous structure 8aCDABCO, the 56membered macrocyclic product 15a $\subset$ DABCO represents one of the largest macrocycles ever prepared via the Weak-Link Approach. In this structure, the $\mathrm{Zn}$ atoms are separated by a distance of distance of $7.02 \AA$ and are bridged by a DABCO ligand (Figure 2). The structure of $15 \mathrm{a} \subset \mathrm{DABCO}$ is slightly puckered toward the $\mathrm{Zn}$ (porphyrin) centers, leading to a slightly staggered porphyrin-porphyrin geometry. As expected, the Rh-P and $\mathrm{Zn}-\mathrm{Zn}$ distance in 8aCDABCO are comparable to those found for $15 \mathrm{a} \subset \mathrm{DABCO}$ while the $\mathrm{Rh}-\mathrm{Rh}$ distance differs significantly, presumably due to the presence of the four $\mathrm{S}$ atoms which have been incorporated in 15aCDABCO (Table 2).

Contrary to $\mathbf{8 b}, \mathbf{1 5 b}$ was quite soluble in pure THF; however, our efforts to crystallize $\mathbf{1 5 b}$ from THF only yielded microcrystalline powder. Attempts to crystallize 15b using the same conditions as used for $\mathbf{8 b}$ led to the formation of magenta single crystals of $15 \mathbf{c} \subset \mathbf{D A B C O}$, which were confirmed by X-ray diffraction analysis at APS (see Supporting Information). Macrocycle 15c $\subset$ DABCO encapsulates a bridging DABCO ligand between two $\mathrm{Zn}$ porphyrins, flanked by tetrahedral $\mathrm{CuCl}_{2}$ centers, again indicating the oxidation of $\mathrm{Cu}^{\mathrm{I}}$ to $\mathrm{Cu}^{\mathrm{II}}$ via solvent dehalogenation as has been observed previously. As for 8c $\subset$ DABCO, isolation of $15 \mathrm{c} \subset \mathrm{DABCO}$ strongly suggests an open macrocyclic structure for $\mathbf{1 5 b}$ as shown in Scheme 3.

\section{Acyl Transfer Catalytic Experiments}

To demonstrate the ability of the $\mathrm{Zn}^{\mathrm{II}}$-porphyrin moieties in $\mathbf{1 4 a}$ and $\mathbf{1 5 a}$ to act cooperatively in an allosterically controlled fashion, we employed a catalytic acyl transfer reaction that has been shown by Sanders and co-workers to accelerate in the presence of trimetallic Lewis acidic porphyrin assemblies. ${ }^{56} \mathrm{We}$ hypothesized that the pyridylcarbinol and acetylimidazole substrates could be brought together within the cavity of $\mathbf{1 5 a}$ by cofacial $\mathrm{Zn}^{\mathrm{II}}$ metal centers and converted to the products in a catalytic fashion by virtue of their proximity (Figure 3). Furthermore, reactions involving 1-acetylimidazole and differentially substituted $X$ pyridylcarbinol (where $X=2,3$, or 4 ) can be used to evaluate the ability of 15a to act allosterically: only the combination of substrates with the right distance can span the cavity and react at an accelerated rate.

The efficiency of both the "closed" (14a) and "open" (15a) supramolecular catalysts in the acyl transfer reaction were evaluated against a control reaction consisting of the monomer $\mathbf{Z n}$ (TPP) and analogous monomeric $\mathrm{Rh}^{\mathrm{I}}$ complexes 16a and $\mathbf{1 6 b}$ (see Supporting Information). 
For 4-pyridylcarbinol (4-PC), both 14a and 15a significantly accelerate the reaction rate, with the open macrocycle 15a being almost 14 times more active than the monomers and twice as fast as the closed macrocycle 14a (Figure 4a). While 14a is depicted as a rigid entity in Figure 3 , its structure is probably dynamic when in solution and the observed catalytic activity may originate from the conformational flexibility around the $\mathrm{S}$ atoms and the low rotational barrier of the porphyrin ligand about the carbon-sulfur bond in the backbone of the ligand. For 3pyridylcarbinol (3-PC), the background-corrected rate $_{\text {macrocycle }} /$ rate $_{\text {monomers }}$ ratio $\left(\right.$ rate $_{\text {monomers }}=$ rate of the reaction in the presence of the $\left[\mathrm{Rh}^{\mathrm{I}}\right.$ monomer $\left.+\mathbf{Z n}(\mathbf{T P P})\right]$ mixture $)$ and the observed allosteric effect ( rate $_{\mathbf{1 5 a}} /$ rate $_{\mathbf{1 4 a}}$ ) only drops slightly (Figure $4 \mathrm{~b}$ ), suggesting that the cavities of 14a and 15a are still flexible enough to accommodate the change in transition state distance for acyl transfer from acetylimidazole upon binding. For 2-pyridylcarbinol (2$\mathrm{PC}$ ), both the rate macrocycle $/$ rate $_{\text {monomers }}$ ratio and the observed allosteric effect (rate 15a ' rate $_{14 a}$ ) drop significantly with respect to 3-and 4-pyridylcarbinol and are similar to those observed for the monomers (Figure 4c). This may be explained by a simple geometry argument: if 2-pyridylcarbinol is bound to one of the $\mathrm{Zn}$ centers, the carbinol group will be pointed away from the imidazole $\mathrm{N}$-acetyl group bound to the other side, resulting in an unfavorable transition state (in comparison to those for 4-and 3-pyridylcarbinol) for productive acyl transfer.

Our catalytic data offers strong evidence that $\mathrm{Cl}^{-} / \mathrm{CO}$ can act as positive allosteric effectors in a $\mathrm{Rh}^{\mathrm{I}}$-based hemilabile supramolecular catalyst system, enhancing the efficiency of bimolecular acyl transfer reactions when the geometry is optimized. Significantly, these effectors operate cooperatively with each other and are incapable of effecting shape change if added independently to the $\mathrm{Rh}^{\mathrm{I}}$ species. That 2-pyridylcarbinol can be selectively discriminated against 3- and 4-pyridylcarbinol provides an impetus for employing these supramolecular assemblies for chemical sensing and shape-selective recognition when coupled to catalytic processes that provide signal amplification.

\section{Conclusion}

In conclusion, we have developed a coordination chemistry-based synthetic approach for the quantitative preparation of flexible cofacial porphyrin assemblies in which the porphyrins act as functional sites within an allosteric framework that is tunable via modulation of peripheral structure control domains. Importantly, these architectures possess cavities whose sizes can be directly controlled in situ via the introduction of simple, cooperative ancillary ligands that bond to the structure control domains. This capability enables the cofacial porphyrin structures to act as allosteric catalysts capable of discriminating different substrate combinations and selectively transforming them into the desired products, two key steps in developing new biomimetic supramolecular systems. Most notably, our synthetic approach should allow for facile access to a range of systems with tunable cofacial porphyrin-porphyrin distances that are useful in studying distance-dependent electron-transfer phenomena, molecular switches, host-guest interactions, and catalysis. Efforts toward elaboration of these areas are currently underway.

\section{Supplementary Material}

Refer to Web version on PubMed Central for supplementary material.

\section{Acknowledgements}

C.A.M acknowledges the NSF and ARO for support of this research and is grateful for a NIH Director's Pioneer Award. This work was also supported, in part, by the NSF-NSEC program under NSF Award Number EEC-0118025. Portions of this work were performed at the DuPont-Northwestern-Dow Collaborative Access Team (DND-CAT) Synchrotron Research Center located at Sector 5 of the Advanced Photon Source. DND-CAT is supported by the E.I. DuPont de Nemours \& Co., the Dow Chemical Company, the U.S. National Science Foundation through Grant 
DMR-9304725 and the State of Illinois through the Department of Commerce and the Board of Higher Education Grant IBHE HECA NWU 96. ChemMat-CARS Sector 15 is principally supported by the National Science Foundation/ Department of Energy under grant number CHE0087817 and by the Illinois Board of Higher Education. The Advanced Photon Source is supported by the U.S. Department of Energy, Basic Energy Sciences, Office of Science, under Contract No. W-31-109-Eng-38.

\section{References}

1. Cowan JA, Sanders JKM. J Chem Soc, Chem Commun 1985:1213-14.

2. Fletcher JT, Therien MJ. J Am Chem Soc 2000;122:12393-12394.

3. Collman JP, Bencosme CS, Durand RR Jr, Kreh RP, Anson FC. J Am Chem Soc 1983;105:2699-703.

4. Feiters MC, Fyfe MCT, Martinez-Diaz MV, Menzer S, Nolte RJM, Stoddart JF, van Kan PJM, Williams DJ. J Am Chem Soc 1997;119:8119-8120.

5. Yagi S, Yonekura I, Awakura M, Ezoe M, Takagishi T. Chem Commun 2001:557-558.

6. Chang CJ, Yeh CY, Nocera DG. J Org Chem 2002;67:1403-1406. [PubMed: 11846697]

7. Jokic D, Asfari Z, Weiss J. Org Lett 2002;4:2129-2132. [PubMed: 12074649]

8. Chng LL, Chang CJ, Nocera DG. J Org Chem 2003;68:4075-4078. [PubMed: 12737594]

9. Collman JP, Tyvoll DA, Chng LL, Fish HT. J Org Chem 1995;60:1926-31.

10. Chen WH, Yan JM, Tagashira Y, Yamaguchi M, Fujita K. Tetrahedron Lett 1999;40:891-894.

11. Faure S, Stern C, Guilard R, Harvey PD. J Am Chem Soc 2004;126:1253-1261. [PubMed: 14746498] 12. Splan KE, Stern CL, Hupp JT. Inorg Chim Acta 2004;357:4005-4014.

13. Hajjaj F, Yoon ZS, Yoon MC, Park J, Satake A, Kim D, Kobuke Y. J Am Chem Soc 2006;128:46124623. [PubMed: 16594698]

14. Fletcher JT, Therien MJ. J Am Chem Soc 2002;124:4298-4311. [PubMed: 11960459]

15. Kobuke Y. Struct Bonding 2006;121:145-165.

16. Collman JP, Fu L, Herrmann PC, Zhang X. Science 1997;275:949-951. [PubMed: 9020071]

17. Chang CJ, Loh ZH, Shi C, Anson FC, Nocera DG. J Am Chem Soc 2004;126:10013-10020. [PubMed: 15303875]

18. Rosenthal J, Pistorio BJ, Chng LL, Nocera DG. J Org Chem 2005;70:1885-1888. [PubMed: 15730314]

19. Collman JP, Wagenknecht PS, Hutchison JE. Angew Chem, Int Ed Engl 1994;106:1620-39.

20. Rosenthal J, Luckett TD, Hodgkiss JM, Nocera DG. J Am Chem Soc 2006;128:6546-6547. [PubMed: 16704240]

21. Brettar J, Gisselbrecht JP, Gross M, Solladie N. Chem Commun 2001:733-734.

22. Jokic D, Boudon C, Pognon G, Bonin M, Schenk KJ, Gross M, Weiss J. Chem- Eur J 2005; 11:4199_ 4209.

23. Merlau M, Grande WJ, Nguyen ST, Hupp JT. J Mol Cat A: Chem 2000;156:79-84.

24. Merlau ML, del Pilar Mejia M, Nguyen ST, Hupp JT. Angew Chem, Int Ed 2001;40:4239-4242.

25. Heo J, Mirkin CA. Angew Chem, Int Ed 2006;45:941-944.

26. Gianneschi NC, Bertin PA, Nguyen ST, Mirkin CA, Zakharov LN, Rheingold AL. J Am Chem Soc 2003;125:10508-10509. [PubMed: 12940719]

27. Gianneschi NC, Cho SH, Nguyen ST, Mirkin CA. Angew Chem, Int Ed 2004;43:5503-5507.

28. Gianneschi NC, Nguyen ST, Mirkin CA. J Am Chem Soc 2005;127:1644-1645. [PubMed: 15700991]

29. Stryer, L. Biochemistry. 4. W.H. Freeman and Company; New York: 1995.

30. Brothers PJ, Collman JP. Acc Chem Res 1986;19:209-15.

31. Chng LL, Chang CJ, Nocera DG. J Org Chem 2003;68:4075-4078. [PubMed: 12737594]

32. Guilard R, Burdet F, Barbe JM, Gros CP, Espinosa E, Shao J, Ou Z, Zhan R, Kadish KM. Inorg Chem 2005;44:3972-3983. [PubMed: 15907125]

33. Slone RV, Hupp JT. Inorg Chem 1997;36:5422-5423.

34. Dinolfo PH, Hupp JT. Chem Mater 2001;13:3113-3125.

35. Dinolfo PH, Lee SJ, Coropceanu V, Bredas JL, Hupp JT. Inorg Chem 2005;44:5789-5797. [PubMed: 16060631] 
36. Benkstein KD, Stern CL, Splan KE, Johnson RC, Walters KA, Vanhelmont FWM, Hupp JT. Eur J Inorg Chem 2002:2818-2822.

37. Ohsaki K, Konishi K, Aida T. Chem Commun 2002:1690-1691.

38. Tomohiro Y, Satake A, Kobuke Y. J Org Chem 2001;66:8442-8446. [PubMed: 11735523]

39. Sanders JKM. Chem Eur J 1998;4:1378-1383.

40. Pognon G, Boudon C, Schenk KJ, Bonin M, Bach B, Weiss J. J Am Chem Soc 2006;128:3488-3489. [PubMed: 16536500]

41. Gianneschi NC, Masar MS III, Mirkin CA. Acc Chem Res 2005;38:825-837. [PubMed: 16285706]

42. Farrell JR, Mirkin CA, Guzei IA, Liable-Sands LM, Rheingold AL. Angew Chem, Int Ed 1998;37:465-467.

43. Holliday BJ, Mirkin CA. Angew Chem, Int Ed 2001;40:2022-2043.

44. Pangborn AB, Giardello MA, Grubbs RH, Rosen RK, Timmers FJ. Organometallics 1996;15:151820.

45. Mao PCM, Mouscadet JF, Leh H, Auclair C, Hsu LY. Chem Pharm Bull 2002;50:1634-1637. [PubMed: 12499608]

46. Brown AM, Ovchinnikov MV, Stern CL, Mirkin CA. J Am Chem Soc 2004;126:14316-14317. [PubMed: 15521726]

47. Laha JK, Dhanalekshmi S, Taniguchi M, Ambroise A, Lindsey JS. Org Process Res Dev 2003;7:799_ 812.

48. Brown AM, Ovchinnikov MV, Mirkin CA. Angew Chem, Int Ed 2005;44:4207-4209.

49. Tomizaki, K-y; Yu, L.; Wei, L.; Bocian, DF.; Lindsey, JS. J Org Chem 2003;68:8199-8207. [PubMed: 14535804]

50. Dixon FM, Eisenberg AE, Farrell JR, Mirkin CA, Liable-Sands LM, Rheingold AL. Inorg Chem 2000;39:3432-3433. [PubMed: 11196796]

51. Masar MS III, Mirkin CA, Stern CL, Zakharov LN, Rheingold AL. Inorg Chem 2004;43:4693-4701. [PubMed: 15257598]

52. Lucchese B, Humphreys KJ, Lee DH, Incarvito CD, Sommer RD, Rheingold AL, Karlin KD. Inorg Chem 2004;43:5987-5998. [PubMed: 15360248]

53. Tyeklar Z, Richard RR, Wei N, Murthy NN, Zubieta J, Karlin KD. J Am Chem Soc 1994;43:59875988.

54. Wei N, Murthy NN, Tyeklar Z, Karlin KD. Inorg Chem 1994;33:1177-1183.

55. Formed by the reaction between $\left[\mathrm{Rh}(\mathrm{NBD}) \mathrm{Cl}_{2}\right.$ and $\mathrm{AgBF}_{4}(\mathrm{NBD}=$ norbornadiene).

56. Mackay LG, Wylie RS, Sanders JKM. J Am Chem Soc 1994;116:3141-3142. 

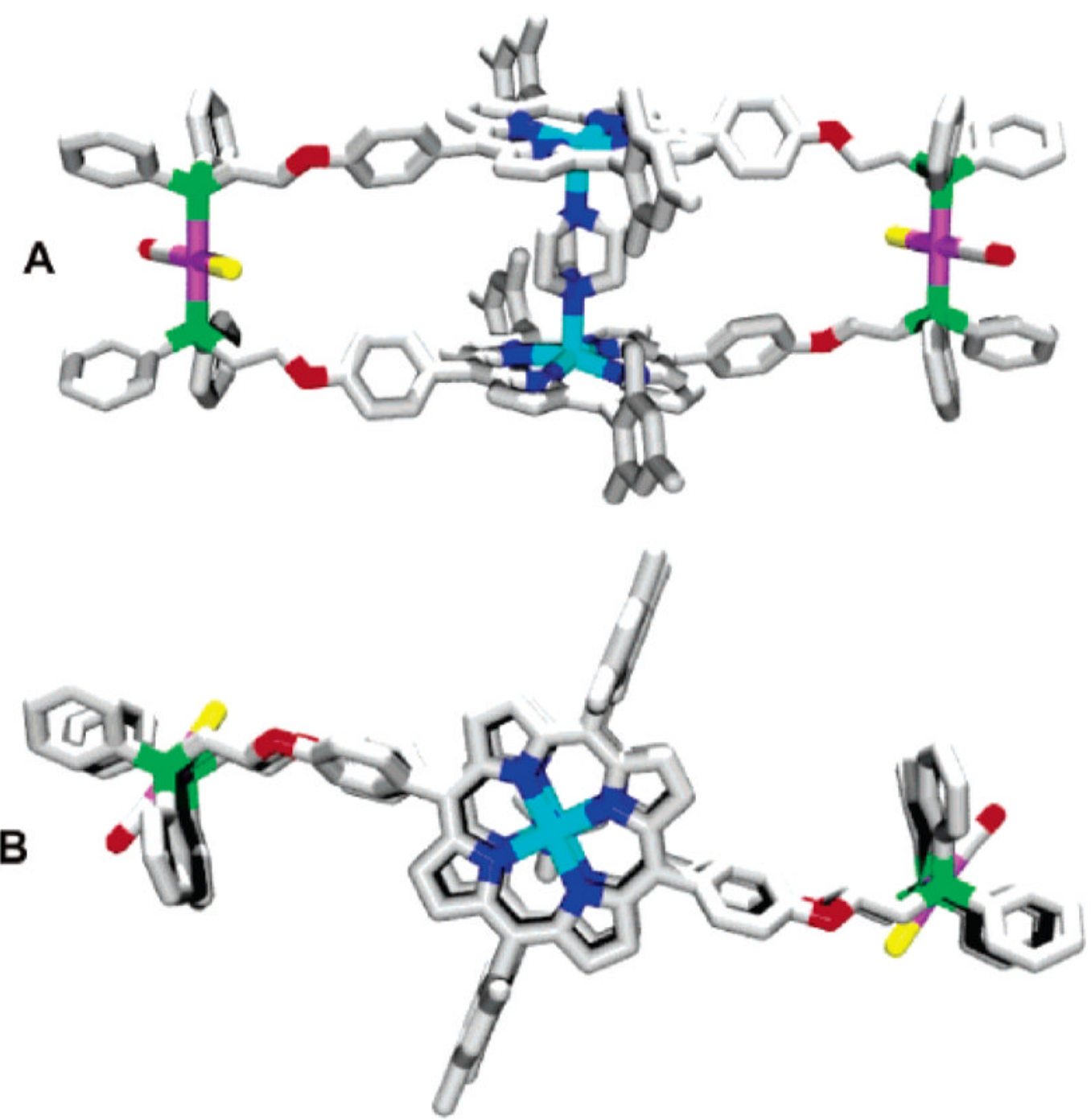

Figure 1.

Graphical representations of the X-ray crystal structure of $8 \mathbf{a} C \mathbf{D A B C O}$ as viewed (A) from the side and (B) from the top containing a molecule of DABCO bridging both $\mathrm{Zn}$ atoms. Hydrogen atoms, disordered DABCO carbon atoms, and solvent molecules have been omitted for clarity. Pink $=\mathrm{Rh}$, Red $=\mathrm{O}$, Yellow $=\mathrm{Cl}$, Green $=\mathrm{P}, \mathrm{Blue}=\mathrm{N}$, Light Blue $=\mathrm{Zn}$. 

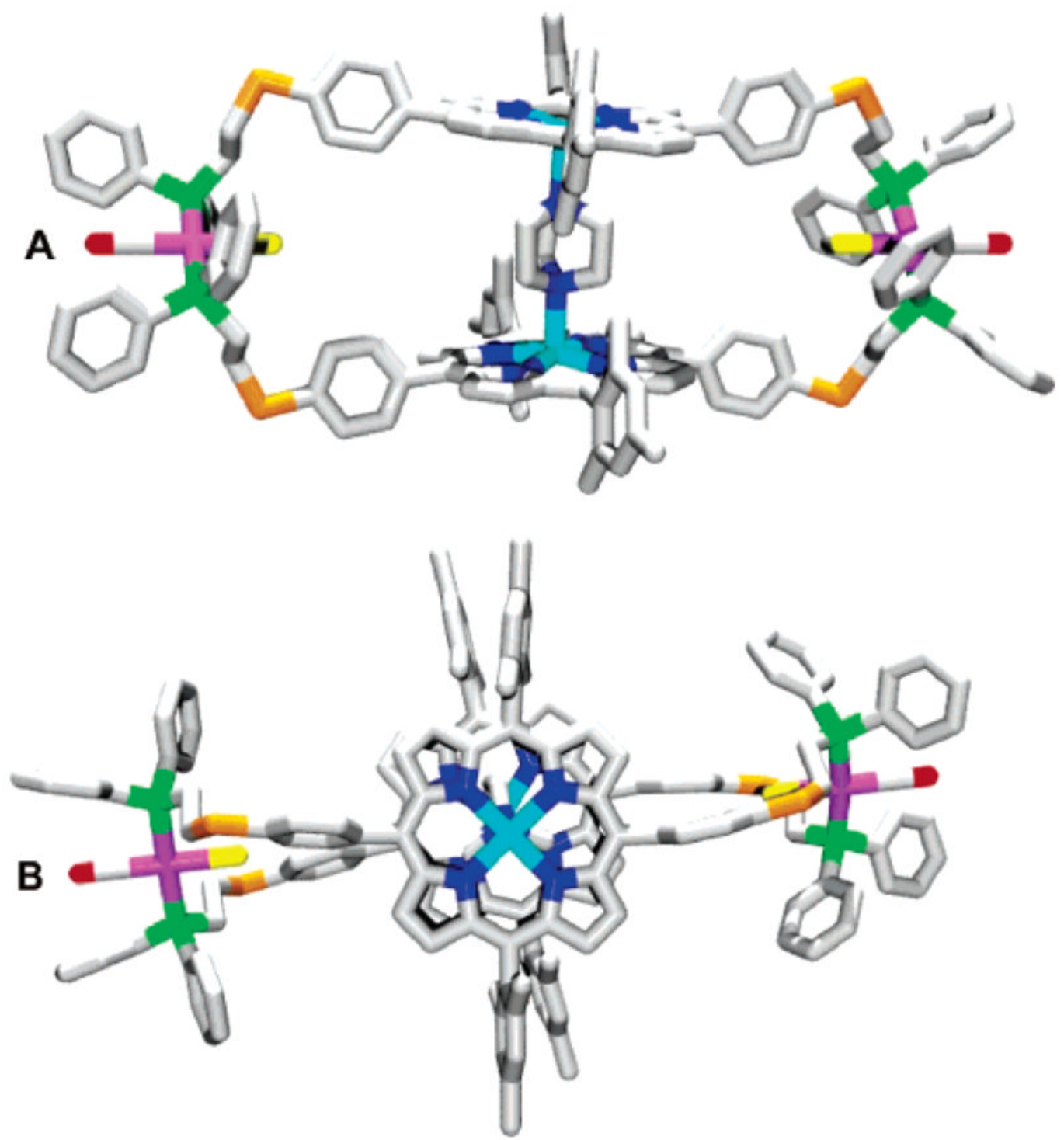

Figure 2.

Graphical representations of the X-ray crystal structure of $\mathbf{1 5 a} \subset \mathbf{D A B C O}$ as viewed (A) from the side and (B) from the top containing a molecule of DABCO bridging both $\mathrm{Zn}$ atoms.

Hydrogen atoms, disordered DABCO carbon atoms and solvent molecules have been omitted for clarity. Gray $=$ Carbon, Pink $=\mathrm{Rh}$, Red $=\mathrm{O}$, Orange $=\mathrm{S}$, Yellow $=\mathrm{Cl}$, Green $=\mathrm{P}$, Dark Blue $=$ N, Light Blue $=$ Zn . 

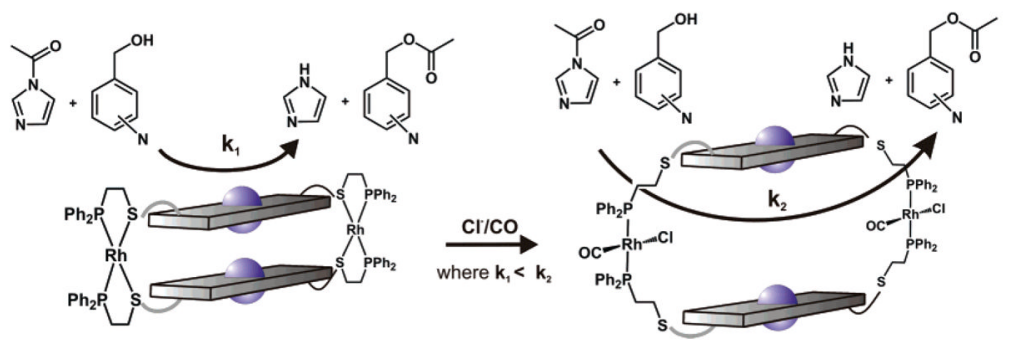

Figure 3.

Acyl transfer reactions catalyzed by (left) a closed macrocycle vs (right) the corresponding open macrocycle. The open macrocycle can preorganize the substrates within the cavity, thereby increasing the rate of the reaction $\left(k_{2}\right)$ in comparison to that $\left(k_{1}\right)$ observed in the presence of the closed macrocycle. 

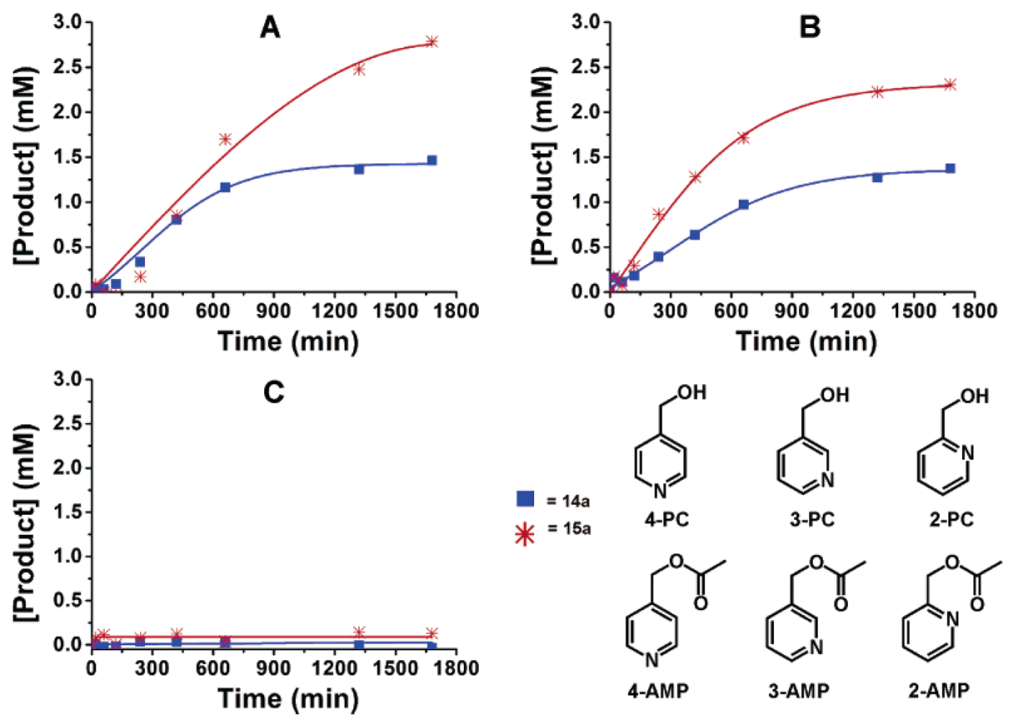

Figure 4.

Formation of the three $X$-(acetoxymethyl)pyridine $(X=2,3$, or 4$)$ isomers by an acyl transfer reaction between 1 -acetylimidazole and $X$-pyridylcarbinol, as catalyzed by $Z$ n-porphyrin complexes 14a and 15a. Concentration vs time plots are shown for the formation of 4(acetoxymethyl)-pyridine (A, 4-AMP), 3-(acetoxymethyl)pyridine (B, 3-AMP), and 2(acetoxymethyl)pyridine (C, 2-AMP). All data were corrected for background reactions (see Supporting Information). Conditions: $\mathrm{CH}_{2} \mathrm{Cl}_{2}$, rt, $9 \mathrm{mM}$ X-pyridylcarbinol, $6 \mathrm{mM} \mathrm{1-}$ acetylimidazole, $2.5 \mathrm{mM}$ biphenyl (GC reference standard), and $0.3 \mathrm{mM}$ supramolecular catalyst (14a and 15a). CO (1 atm) and appropriate amounts of benzyltriethylammonium chloride when indicated. 


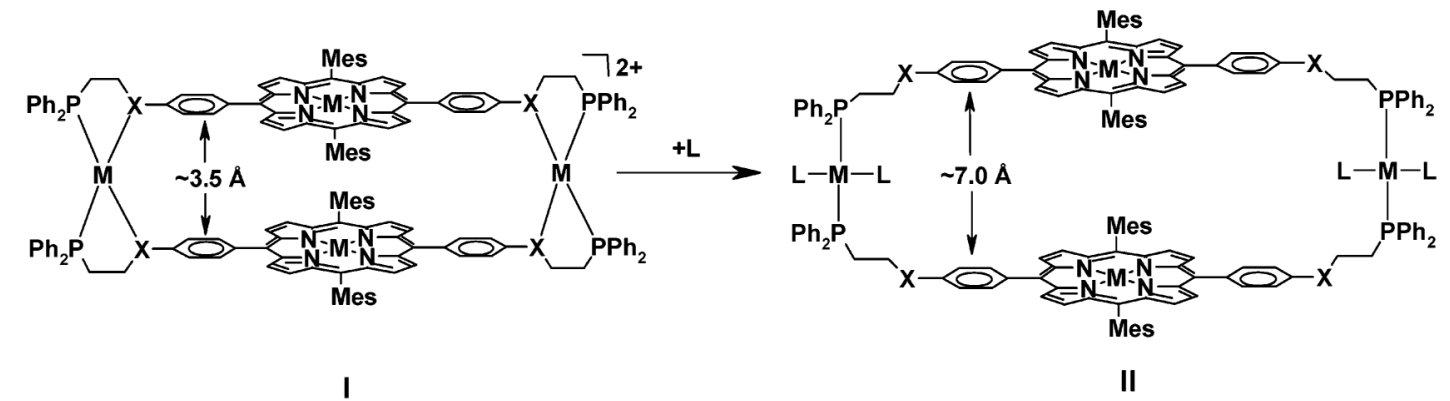

Scheme 1. Design of Allosteric Porphyrin-Based Supramolecules Whose Cavity Sizes Can Be Modified by the Binding of a Ligand $\mathrm{L}^{a}$

${ }^{a} \mathbf{I}$ : Condensed Macrocycle, II: Open Macrocycle. $\mathrm{PPh}_{2}=$ diphenylphosphine and MES = 1,3,5-trimethylbenzene. 

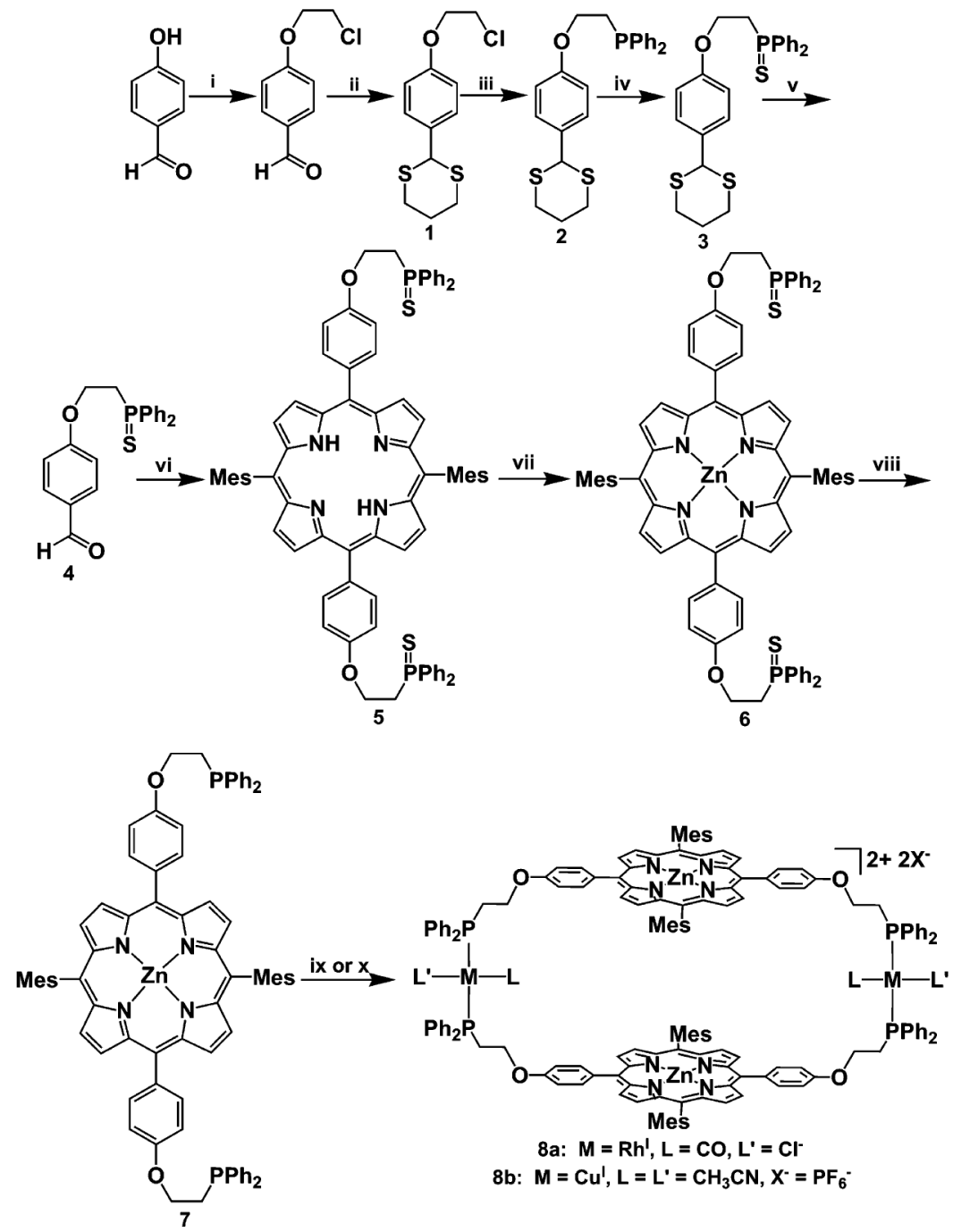

Scheme 2. Synthesis of Ether-Based Ligand 7 and Macrocycles $8 \mathrm{a}$ and $8 \mathrm{~b}^{\boldsymbol{a}}$

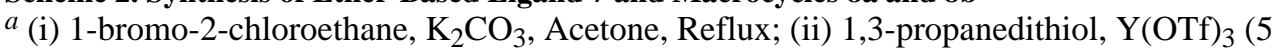
mol \%), $\mathrm{CH}_{3} \mathrm{CN}$; (iii) $\mathrm{KPPh}_{2}$, THF; (iv) $\mathrm{S}_{8}$, THF; (v) $\mathrm{NaNO}_{2}, \mathrm{AcCl} / \mathrm{H}_{2} \mathrm{O}, \mathrm{CH}_{2} \mathrm{Cl}_{2}, 0{ }^{\circ} \mathrm{C} \rightarrow \mathrm{rt}$; (vi) 5-mesityldipyrromethane, $\mathrm{BF}_{3} \bullet \mathrm{OEt}_{2}, \mathrm{DDQ}, \mathrm{NEt}_{3}, \mathrm{CHCl}_{3}, 4 \AA$ Molecular Sieves; (vii) $\mathrm{Zn}$ $(\mathrm{OAc})_{2} \cdot 2 \mathrm{H}_{2} \mathrm{O}, 4: 1 \mathrm{CHCl}_{3} / \mathrm{MeOH}$, Reflux; (viii) $\mathrm{Cp}_{2} \mathrm{ZrHCl}$, THF, $60{ }^{\circ} \mathrm{C}$; (ix) $[\mathrm{Rh}$ $\left.(\mathrm{CO})_{2}(\mathrm{Cl})\right]_{2}, \mathrm{CH}_{2} \mathrm{Cl}_{2} / \mathrm{THF}$; (x) $\left[\mathrm{Cu}\left(\mathrm{CH}_{3} \mathrm{CN}\right)_{4}\right] \mathrm{PF}_{6}, \mathrm{CH}_{2} \mathrm{Cl}_{2} / \mathrm{THF}$. 


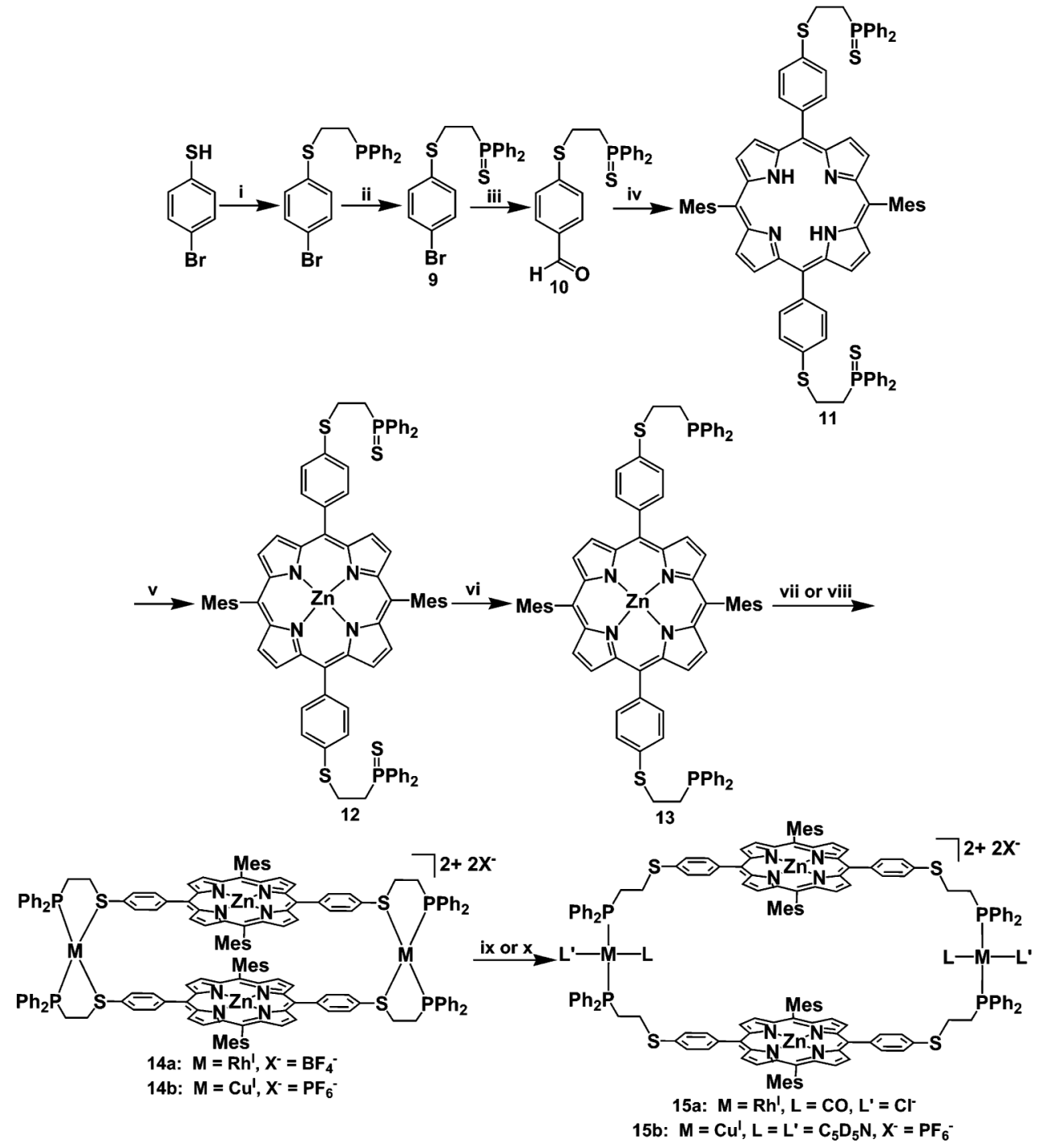

Scheme 3. Synthesis of Thioether-Based Ligand 13 and Macrocycles 14a-b, 15a-b ${ }^{a}$

$a$ (i) $\mathrm{ClCH}_{2} \mathrm{CH}_{2} \mathrm{PPh}_{2}, \mathrm{Cs}_{2} \mathrm{CO}_{3}, \mathrm{CH}_{3} \mathrm{CN}$, Reflux; (ii) $\mathrm{S}_{8}$, THF; (iii) $n$-BuLi, DMF, THF, $-78^{\circ}$ $\mathrm{C}$; (iv) 5-mesityldipyrromethane, $\mathrm{BF}_{3} \bullet \mathrm{OEt}_{2}, \mathrm{DDQ}, \mathrm{NEt}_{3}, \mathrm{CHCl}_{3}, 4 \AA$ A Molecular Sieves; (v) $\mathrm{Zn}(\mathrm{OAc})_{2} \cdot 2 \mathrm{H}_{2} \mathrm{O}, 4: 1 \mathrm{CHCl}_{3} / \mathrm{MeOH}$, Reflux; (vi) $\mathrm{Cp}_{2} \mathrm{ZrHCl}$, THF, $60{ }^{\circ} \mathrm{C}$; (vii) for 14a: [Rh (NBD)Cl $]_{2}, \mathrm{AgBF}_{4}, \mathrm{CH}_{2} \mathrm{Cl}_{2} / \mathrm{THF}$; (viii) for 14b: $\left[\mathrm{Cu}\left(\mathrm{CH}_{3} \mathrm{CN}\right)_{4}\right] \mathrm{PF}_{6}, \mathrm{CH}_{2} \mathrm{Cl}_{2} / \mathrm{THF}$; (ix) for 15a: $\mathrm{PPNCl} / \mathrm{CO}$ (1 atm); (x) for $\mathbf{1 5 b}: \mathrm{C}_{5} \mathrm{D}_{5} \mathrm{~N}$. 
Table 1

X-ray Crystallographic Data for 8a $\subset$ DABCO and 15a $\subset$ DABCO

\begin{tabular}{|c|c|c|}
\hline & 8aСDABCO & 15aСDABCO \\
\hline $\begin{array}{l}\text { empirical formula } \\
\text { formula weight } \\
\text { Temperature } \\
\text { Wavelength } \\
\text { crystal system, space group } \\
\text { unit cell dimensions } \\
\\
\text { volume } \\
Z \text {, calculated density } \\
\text { absorption coefficient } \\
F(000) \\
\text { crystal size } \\
\text { theta range for data collection } \\
\text { reflections collected/unique } \\
\text { absorption correction } \\
\text { max. and min. transmission } \\
\text { refinement method } \\
\text { data/restraints/parameters } \\
\text { goodness-of-fit on } \mathrm{F}^{\wedge} 2 \\
\text { final } R \text { indices }[I>2 \text { sigma }(I)] \\
R \text { indices (all data) } \\
\text { largest diff. peak and hole }\end{array}$ & $\begin{array}{l}\mathrm{C}_{181} \mathrm{H}_{166} \mathrm{Cl}_{4} \mathrm{~N}_{12} \mathrm{O}_{8} \mathrm{P}_{4} \mathrm{Rh}_{2} \mathrm{Zn}_{2} \\
3239.5 \\
153(2) \mathrm{K} \\
0.71073 \AA \\
\text { triclinic, } P \overline{1} \\
a=13.9395(13) \AA \alpha=83.044(2)^{\circ} \\
b=17.1623(17) \AA \beta=73.368(2)^{\circ} \\
c=20.894(2) \AA \gamma=74.250(2)^{\circ} \\
4604.5(8) \AA^{3} \\
1,1.168 \mathrm{Mg}^{-} \mathrm{m}^{3} \\
0.581 \mathrm{~mm}^{3} \\
1678 \\
0.170 \times 0.170 \times 0.50 \mathrm{~mm} \\
1.02 \text { to } 28.83^{\circ} \\
42335 / 21291[R(\text { int })=0.0712] \\
\text { integration } \\
0.9695 \text { and } 0.8265 \\
\text { full-matrix least-squares on } F^{2} \\
21291 / 0 / 915 \\
0.912 \\
R 1=0.0910, w R 2=0.2301 \\
R 1=0.1924, w R 2=0.2591 \\
1.378 \text { and }-0.747 \mathrm{e}^{-} / \AA^{-3}\end{array}$ & $\begin{array}{l}\mathrm{C}_{172} \mathrm{H}_{142} \mathrm{Cl}_{2} \mathrm{~N}_{10} \mathrm{O}_{4} \mathrm{P}_{4} \mathrm{Rh}_{2} \mathrm{~S}_{4} \mathrm{Zn}_{2} \\
3072.54 \\
100(2) \mathrm{K} \\
0.48595 \AA \\
\text { monoclinic, } C 2 / c \\
a=17.0086(10) \AA \\
b=44.661(3) \AA{ }^{\circ}=104.252(2)^{\circ} \\
c=23.0053(13) \AA \\
16937.3(17) \AA^{3} \\
4,1.205 \mathrm{Mg}^{3} / \mathrm{m}^{3} \\
0.342 \mathrm{~mm}^{-1} \\
6336 \\
0.150 \times 0.025 \times 0.010 \mathrm{~mm} \\
0.90 \text { to } 15.70^{\circ} \\
123297 / 12229[R(\text { int })=0.0780] \\
\text { empirical } \\
1.000000 \text { and } 0.649500 \\
\text { full-matrix least-squares on } F^{2} \\
12229 / 0 / 921 \\
1.063 \\
R 1=0.0937, w R 2=0.2816 \\
R 1=0.1199, w R 2=0.3081 \\
2.236 \text { and }-0.800 \mathrm{e}^{-} / \AA^{-3}\end{array}$ \\
\hline
\end{tabular}


Table 2

Selected Distances for $\mathbf{8 a} \subset \mathbf{D A B C O}$ and $15 \mathbf{a} \subset \mathbf{D A B C O}$

\begin{tabular}{lcc}
\hline selected distance & 8aCDABCO $(\AA)$ & 15aCDABCO $(\AA)$ \\
\hline $\mathrm{Rh}-\mathrm{Rh}$ & 24.8 & 22.5 \\
$\mathrm{Rh}-\mathrm{P}$ & $2.32,2.31$ & 2.30 \\
$\mathrm{Zn}-\mathrm{Zn}$ & 7.09 & 7.02 \\
\hline
\end{tabular}

